\title{
WAVE PROPAGATION IN LAYERED DRY, SATURATED AND UNSATURATED POROELASTIC MEDIA
}

\author{
G. DEGRANDE, $\uparrow$ G. DE ROECK, P. VAN DEN BROECK \\ K.U. Leuven, Department of Civil Engineering, W. de Croylaan 2, B-3001 Heverlee, Belgium \\ and \\ D. SMEULDERS \\ T.U. Delft, Faculty of Applied Earth Sciences, P.O. Box 5028, NL-2600 GA Delft, The \\ Netherlands
}

(Received 9 June 1997; in revised form 25 October 1997)

\begin{abstract}
An exact stiffness formalism is presented to study harmonic and transient wave propagation in multilayered dry, saturated and unsaturated isotropic poroelastic media. Smeulders' extension of Biot's poroelastic theory is used to incorporate unsaturated porous media with a small gas fraction. A wide range of problems in geophysical and civil engineering can be treated, ranging from amplification of plane harmonic waves, dispersion and attenuation of surface waves to transient wave propagation due to a forced excitation. The effect of full or partial saturation on wave propagation in a poroelastic layered halfspace is demonstrated in a numerical example, in which the layering is caused by a moving ground water table. (C) 1998 Elsevier Science. All rights reserved.
\end{abstract}

\section{INTRODUCTION}

Many materials encountered in civil, geophysical and biomechanical engineering can be considered as porous media consisting of an assemblage of solid particles and a pore space. The pore space may be filled with air (dry medium), a fluid (saturated medium) or both (unsaturated medium).

The study of wave propagation in layered media has received considerable attention, especially in the context of exploration geophysics, seismology and engineering. The restriction to linear problems defined on horizontally layered media allows the use of integral transforms and the formulation of a layer and halfspace stiffness matrix that can be used in an exact stiffness formalism (Kausel and Roesset, 1981). In spite of the aforementioned restrictions, this formalism allows for the solution of various important problems such as site amplification of plane harmonic waves, dispersion and attenuation of surface waves and harmonic and transient wave propagation due to a forced excitation.

Whereas the original contributions have been formulated within the frame of classical elastodynamics, treating the medium as a monophasic continuum, the importance of the interaction between the pore fluid and the solid skeleton is now generally recognized. The dynamic behaviour of saturated porous media is described by Biot's poroelastic equations (Biot, 1956). Using this theory, Deresiewicz (1962) and Jones (1961) have studied the propagation of free surface waves in a saturated poroelastic halfspace while Paul (1976a; b) and Philippacopoulos (1988a) have considered transient waves. Philippacopoulos (1988b) has been the first to study the case of a "partially" saturated poroelastic halfspace where a dry layer is on top of a saturated halfspace, representing the case of a moving water table.

By analogy with the monophasic elements, Degrande (1992) and Degrande and De Roeck $(1992,1993)$ have formulated the dynamic saturated layer and halfspace stiffness

$\dagger$ Author to whom correspondence should be addressed. Tel. : 0032163216 67. Fax: 003216321988. Email : geert.degrande@bwk.kuleuven.ac.be. 
matrices. These matrices can be used together with their dry counterpart in an exact stiffness formalism to model harmonic and transient wave propagation in dry and saturated poroelastic media. Similar work has recently been reported by Rajapakse and Senjuntichai (1995), who succeeded in deriving analytical expressions for the elements of the dynamic stiffness matrices of the saturated poroelastic layer and halfspace element, thus allowing a more efficient implementation in a computer program.

Whereas the mathematical formulation of wave propagation in saturated porous media has been quite successful, it should be emphasized that in practice, the presence of small amounts of gas is not unlikely at all, as porous media will hardly ever be fully liquidsaturated. One might envisage small gas remnants in oil saturated geological strata, or air bubbles trapped in marine sediments. Based on shock tube experiments, Smeulders (1992) has clearly demonstrated how small amounts of gas in the pores of a saturated porous medium affect the one-dimensional wave propagation characteristics. He has also confirmed theoretically his experimental findings by a modification of Biot's poroelastic theory.

The question now arises how a small amount of air bubbles affects wave propagation in two- or three-dimensional poroelastic media. Therefore, the topics covered in this paper are as follows. Firstly, the equations governing the dynamic response of dry, saturated and unsaturated isotropic poroelastic media will be briefly reviewed. Secondly, it will be demonstrated how an exact stiffness formulation for wave propagation in isotropic dry and saturated layered poroelastic media can be modified to incorporate Smeulders' modification of Biot's poroelastic theory to account for the presence of a small amount of air bubbles. Thirdly, we will demonstrate by means of a numerical example how full and partial saturation influences harmonic and transient wave propagation in an axisymmetric poroelastic layered halfspace, where the layering is due to a water table at a depth $H$ below the free surface.

\section{GOVERNING EQUATIONS FOR ISOTROPIC MEDIA}

A porous medium consists of a solid skeleton and a pore space that may be filled with air, a fluid or both. The pore space is connected, enabling the filtration of the pore fluid through the porous medium. The solid skeleton consists of the solid matrix and the empty connected pore space.

In the following, the equations that describe the propagation of waves in isotropic dry, saturated and unsaturated media will be briefly reviewed. We have intentionally restricted the formulation to the isotropic case in order to reduce the mathematical complexity. A full anisotropic version of the formalism would be useful, however, for the study of wave propagation in layered sediments that often appear to be anisotropic.

\subsection{Dry poroelastic medium}

The dynamic behaviour of a dry porous medium or single phase medium can be described within the frame of classical continuum mechanics. The displacement vector in the solid skeleton is denoted by $\mathbf{u}^{s}$. The small strain tensor $\boldsymbol{\varepsilon}^{s}$ in the solid skeleton is equal to the symmetric part of the solid skeleton displacement gradient as :

$$
\varepsilon_{i j}^{s}=\frac{1}{2}\left(u_{i, j}^{s}+u_{j, i}^{s}\right) .
$$

The equilibrium equation of the dry porous medium is :

$$
\sigma_{i j, j}+\rho^{s} b_{i}=\rho^{s} \ddot{u_{i}^{s}} .
$$

$\boldsymbol{\sigma}$ is the stress tensor, $\rho^{s} \mathbf{b}$ is the body force vector and $\rho^{s}=\rho_{s}(1-n)$ is the density of the solid skeleton, with $\rho_{s}$ the density of the solid grains and $n$ the porosity. A superimposed dot on a variable denotes differentiation with respect to time. We assume that convective terms can be discarded.

The constitutive equations for an isotropic linear elastic material are: 


$$
\sigma_{i j}=2 \mu^{s} \varepsilon_{i j}^{s}+\lambda^{s} \varepsilon_{k k}^{s} \delta_{i j}
$$

with $\mu^{s}$ and $\lambda^{s}$ the Lamé coefficients and $\delta_{i j}$ the Kronecker delta. In the following derivations in the frequency domain, it will be implicitly assumed that the Lamé coefficients are complex numbers by application of the correspondence principle, i.e. multiplication of $\mu^{s}$ and $\lambda^{s}+2 \mu^{s}$ by $\left(1+2 i \beta_{s}^{s}\right)$ and $\left(1+2 i \beta_{p}^{s}\right)$, respectively. Here, $i=\sqrt{-1}$ is the imaginary unit and $\beta_{s}^{s}$ and $\beta_{p}^{s}$ are the hysteretic material damping ratios for rotational and dilatational deformation, respectively.

\subsection{Saturated poroelastic medium}

In a saturated porous medium, the connected pore space is entirely filled with a fluid. The small strain dynamic behaviour of a saturated porous medium can be described with Biot's poroelastic theory (Biot, 1956).

The displacement vectors in both phases of a saturated porous medium are denoted by $\mathbf{u}^{\alpha}$ where $\alpha=s$ for the solid skeleton and $\alpha=f$ for the pore fluid. The fluid flow relative to the solid skeleton measured in terms of volume per unit area of the bulk medium is equal to $\mathbf{w}=n\left(\mathbf{u}^{f}-\mathbf{u}^{s}\right)$. The relation (1) is supplemented with the following expression for the volume of fluid $\zeta$ which escapes from the pores of a unit volume of bulk material:

$$
\zeta=w_{i, i}
$$

The global equilibrium equation of the saturated porous medium is:

$$
\sigma_{i j, j}+\rho b_{i}=\rho \ddot{u}_{i}^{s}+\rho_{f} \ddot{w}_{i} .
$$

Here, $\boldsymbol{\sigma}$ is the total stress tensor, $\rho \mathbf{b}$ is the body force vector, $\rho=\rho_{f} n+\rho_{s}(1-n)$ is the mixture density and $\rho_{f}$ is the density of the pore fluid. The motion of the pore fluid with respect to the solid skeleton can be described by the generalized Darcy's law (Biot, 1956; Coussy, 1995) as :

$$
-p_{, i}+\rho_{f} b_{i}=\rho_{f} \ddot{u}_{i}^{s}+\frac{\rho_{f} a_{i j}}{n} \ddot{w}_{j}+\frac{1}{n^{2}} \xi_{i j} \dot{w}_{j}
$$

where $p$ is the pore fluid pressure. The symmetric tensor $a_{i j}$ accounts for the tortuosity of the pores and reduces to $a \delta_{i j}$ in the isotropic case. The viscous interaction between the pore fluid and the solid skeleton is represented by a symmetric positive definite second order tensor $\boldsymbol{\xi}$. In the frequency domain, this tensor will be written as $\xi(\omega)=F(\omega) \xi_{0} . F(\omega)$ is a complex frequency dependent viscosity correction factor that describes the transition behaviour from viscosity dominated flow in the low frequency regime towards inertia dominated flow at high frequencies (Auriault et al., 1985; Johnson et al., 1987; Smeulders, 1992; Smeulders et al., 1992). The tensor $\boldsymbol{\xi}_{0}$ is related to the Darcy permeability tensor $\mathbf{k}_{0}$ by $\xi_{0}=n^{2} \gamma_{f} \mathbf{k}_{0}^{-1}$ and to the specific permeability tensor $\mathbf{k}_{I 0}$ by $\boldsymbol{\xi}_{0}=n^{2} \eta_{d} \mathbf{k}_{I 0}^{-1}$. Herein, $\gamma_{f}$ and $\eta_{d}$ are the specific weight and dynamic viscosity of the pore fluid. For an isotropic poroelastic medium, the tensors $\xi_{0 i j}, k_{0 i j}$ and $k_{I 0 i j}$ reduce to $\xi_{0} \delta_{i j}, k_{0} \delta_{i j}$ and $k_{I 0} \delta_{i j}$, respectively.

Finally, the isotropic poroelastic constitutive equations read:

$$
\sigma_{i j}=2 \mu^{s} \varepsilon_{i j}^{s}+\lambda^{s} \varepsilon_{k k}^{s} \delta_{i j}-\alpha p \delta_{i j} ; \quad-p=\alpha M \varepsilon_{k k}^{s}-M \zeta,
$$

with the Biot coefficients $\alpha$ and $M$ defined as:

$$
\alpha=1-\frac{K_{d}}{K_{s}} ; \quad \frac{1}{M}=\frac{n}{K_{f}}+\frac{\alpha-n}{K_{s}} .
$$

$K_{d}, K_{s}$ and $K_{f}$ are the bulk moduli of the drained solid skeleton, the solid matrix, and the 
pore fluid, respectively. The Biot coefficients are subject to the thermodynamical restrictions $M \geqslant 0$ and $n \leqslant \alpha \leqslant 1$.

\subsection{Unsaturated poroelastic medium}

In an unsaturated porous medium, the connected pore space is filled with a liquid and a gas phase. We follow Smeulders (1992) assuming that the gas fraction in the pores is small so that the gas bubbles can be considered as part of the pore fluid. Consequently, the dynamic behaviour of an unsaturated porous medium with a small gas fraction can be described by Biot's two-phase theory for saturated porous media if the influence of the gas phase on the compressibility of the fluid phase is accounted for. The bulk modulus $K_{f}$ of the "generalized" pore fluid is :

$$
\frac{1}{K_{f}}=\frac{1-g}{K_{l}}+\frac{g}{K_{g}},
$$

where $g$ denotes the pore gas fraction. $K_{l}$ is the liquid bulk modulus and $K_{g}$ the effective bulk modulus of the gas phase, which relates the averaged bubble volume $V_{g}$ to a change in liquid pressure $p_{\infty}$ far away from the bubble :

$$
\frac{1}{K_{g}}=-\frac{1}{V_{g}} \frac{\partial V_{g}}{\partial p_{\infty}} .
$$

Gas bubbles in liquid can vibrate and have a fundamental resonance frequency. Moreover, several damping mechanisms result in a phase shift between a change in the gas bubble volume and the fluid pressure far away from the gas bubble. In the frequency domain, this can be described by a complex effective bulk modulus $K_{g}$ of the gas phase. We assume that the shape of the gas bubbles is spherical, that in the case of small gas fractions the medium outside the bubble can be considered as saturated, and that, since the bubble motion is considerably larger than the motion of the solid skeleton, the solid skeleton is rigid. This yields the following expression for the effective bulk modulus $K_{g}$ of the gas phase (Smeulders, 1992):

$$
K_{g}=\left(n_{p} p_{g 0}-\frac{2}{3} \frac{\sigma}{R_{0}}\right)+\frac{4}{3} \eta_{d} i \omega-\frac{\frac{1}{3} a \rho_{f} R_{0}^{2}}{1+i k_{r e} R_{0}}\left(\omega^{2}-i \omega \omega_{c} F\right),
$$

with $\omega$ the angular frequency, $\omega_{c}$ a characteristic frequency defined as $\eta_{d} n / \rho_{f} k_{I 0} a$ (Biot, 1956; Smeulders, 1992), $R_{0}$ the bubble radius, $n_{p}$ the complex polytropic constant, $p_{g 0}$ the gas bubble pressure, $\sigma$ the surface tension, $k_{r e}=\sqrt{a} / C_{f} \sqrt{\omega^{2}-i \omega \omega_{c} F}$ the effective wavenumber, corresponding to the propagation of the second Biot wave in the limiting case of a stiff frame, and $C_{f}$ the speed of sound in the fluid. Zero subscribed quantities refer to a fixed reference equilibrium state. The effective bulk modulus of the gas phase is a complex frequency dependent number. Energy dissipation due to Darcy flow, acoustic, thermal and viscous damping is incorporated and has been discussed in much detail by Smeulders (1992) and Smeulders et al. (1992). Thermal coupling is due to heat exchange between the vibrating gas bubbles and the surrounding porous matrix, while acoustic damping originates from the oscillating gas bubbles emitting spherical sound waves into the surrounding pore fluid. Darcy flow and viscous damping mechanisms evolve from fluid-grain interactions and fluid velocity gradients, respectively.

\subsection{Displacement equations of motion}

From the previous discussion it follows that the dynamic behaviour of saturated and unsaturated poroelastic media may be described by means of Biot's poroelastic theory. In the unsaturated case, proper account should be made of the influence of a small amount of 
gas on the compressibility of the liquid-gas mixture that saturates the pores. Using the poroelastic constitutive eqns (7) and the linearized strain-displacement relations (1) and (4), the equilibrium eqns (5) and (6) can be written in terms of the displacement vectors $\mathbf{u}^{s}$ and $\mathbf{w}$ as :

$$
\begin{aligned}
\mu^{s} \nabla \cdot \nabla \mathbf{u}^{s}+\left(\lambda^{s}+\mu^{s}+\alpha^{2} M\right) \nabla \nabla \cdot \mathbf{u}^{s}+\alpha M \nabla \nabla \cdot \mathbf{w}+\rho \mathbf{b} & =\rho \ddot{\mathbf{u}}^{s}+\rho_{f} \ddot{\mathbf{w}}, \\
\alpha M \nabla \nabla \cdot \mathbf{u}^{s}+M \nabla \nabla \cdot \mathbf{w}+\rho_{f} \mathbf{b} & =\rho_{f} \ddot{\mathbf{u}}^{s}+\frac{\rho_{f} a}{n} \ddot{\mathbf{w}}+\frac{\xi}{n^{2}} \dot{\mathbf{w}} .
\end{aligned}
$$

\subsection{In-plane and out-of-plane motion}

In a Cartesian coordinate system with unit vectors $\mathbf{e}_{x}, \mathbf{e}_{y}$ and $\mathbf{e}_{z}$, the displacement vectors $\mathbf{u}^{s}$ and $\mathbf{w}$ and decomposed in terms of the scalar wave potentials $\Phi^{\alpha}, \Psi^{\alpha}$ and $\chi^{\alpha}$ (Atkin, 1968 ; Eringen and Suhubi, 1975) as :

$$
\begin{aligned}
\mathbf{u}^{s} & =\nabla \Phi^{s}+\nabla \times \Psi^{s} \mathbf{e}_{y}+\nabla \times \chi^{s} \mathbf{e}_{z}, \\
\mathbf{w} & =\nabla \Phi^{f}+\nabla \times \Psi^{f} \mathbf{e}_{y}+\nabla \times \chi^{f} \mathbf{e}_{z} .
\end{aligned}
$$

This specific form of the Helmholtz decomposition has been chosen to decouple immediately the in-plane motions in the $(x, z)$-plane from the out-of-plane motions in the $y$-direction, with $x$ the horizontal axis parallel to the free surface, $z$ the vertical axis normal to the free surface and $y$ the horizontal axis normal to the $(x, z)$-plane. After substitution of the decomposition (13) in the displacement equations of motion (12), the following set of uncoupled hyperbolic partial differential equations (PDE) is obtained:

$$
\begin{gathered}
{\left[-\mathbf{K}_{p} \nabla^{2}+\mathbf{M} \frac{\partial^{2}}{\partial t^{2}}+\mathbf{E} \frac{\partial}{\partial t}\right]\left\{\begin{array}{l}
\left.\Phi^{s}\right\} \\
\Phi^{f}
\end{array}\right\}=\left\{\begin{array}{l}
0 \\
0
\end{array}\right\},} \\
{\left[-\mathbf{K}_{s} \nabla^{2}+\mathbf{M} \frac{\partial^{2}}{\partial t^{2}}+\mathbf{E} \frac{\partial}{\partial t}\right]\left\{\begin{array}{l}
\Psi^{s} \\
\Psi^{f}
\end{array}\right\}=\left\{\begin{array}{l}
0 \\
0
\end{array}\right\},} \\
{\left[-\mathbf{K}_{s} \nabla^{2}+\mathbf{M} \frac{\partial^{2}}{\partial t^{2}}+\mathbf{E} \frac{\partial}{\partial t}\right]\left\{\begin{array}{l}
\chi^{s} \\
\chi^{f}
\end{array}\right\}=\left\{\begin{array}{l}
0 \\
0
\end{array}\right\} .}
\end{gathered}
$$

The first two equations describe in-plane motion in terms of the scalar wave potentials $\Phi^{\alpha}$ (P or longitudinal waves) and $\Psi^{\alpha}$ (SV or vertically polarized shear wave), while the third describes out-of-plane motion as a function of the scalar potentials $\chi^{\alpha}$ (SH or horizontally polarized shear wave). The following discussion will be restricted to the propagation of inplane $\mathrm{P}$ and $\mathrm{SV}$ waves. The dilatational and rotational stiffness matrix are defined as:

$$
\mathbf{K}_{p}=\left[\begin{array}{cc}
\lambda^{s}+2 \mu^{s}+\alpha^{2} M & \alpha M \\
\alpha M & M
\end{array}\right] ; \quad \mathbf{K}_{s}=\left[\begin{array}{cc}
\mu^{s} & 0 \\
0 & 0
\end{array}\right],
$$

and the mass and damping matrix are equal to:

$$
\mathbf{M}=\left[\begin{array}{cc}
\rho & \rho_{f} \\
\rho_{f} & \rho_{f} a / n
\end{array}\right] ; \quad \mathbf{E}=\left[\begin{array}{cc}
0 & 0 \\
0 & \xi / n^{2}
\end{array}\right] .
$$

The elastodynamical behaviour of a medium can also be represented in cylindrical coordinates $(r, z, \theta)$ where $r$ and $\theta$ are the radial and circumferential coordinate, respectively. This form of the equations is useful for example for applications involving a borehole. 


\section{DISPERSION RELATIONS}

\subsection{Dilatational waves}

The in-plane propagation of dilatational $(\mathrm{P})$ waves in porous media is described by the first linear hyperbolic PDE in eqn (14). A Fourier transformation of the time $t$ to the frequency $\omega$ is followed by a Fourier transformation of the horizontal coordinate $x$ to the horizontal wavenumber $k_{x}$, as it is assumed that the geometry is invariant in the horizontal $x$-direction. This results in the following system of linear coupled ordinary differential equations (ODE) in the wavenumber-frequency domain:

$$
\left[\left(k_{x}^{2}-\frac{\mathrm{d}^{2}}{\mathrm{~d} z^{2}}\right) \mathbf{K}_{p}-\omega^{2} \mathbf{M}+i \omega \mathbf{E}\right]\left\{\begin{array}{l}
\tilde{\Phi}^{s} \\
\tilde{\Phi}^{f}
\end{array}\right\}=\left\{\begin{array}{l}
0 \\
0
\end{array}\right\} .
$$

Here, a tilde above a variable denotes its representation in the wavenumber-frequency domain. As the only independent variable in the ODE (17) is the vertical coordinate $z$, the following analytical solutions can be proposed for the wave potentials :

$$
\tilde{\Phi}^{\alpha}\left(k_{x}, z, \omega\right)=P^{\alpha} \exp \left(-i k_{z p} z\right)
$$

where $k_{z p}$ is the vertical component of the dilatational wave propagation vector $\mathbf{k}_{p}\left(k_{x}, k_{z p}\right)$. The eigenvector components $P^{\alpha}$ depend on the boundary and initial conditions of the problem. Setting the solutions (18) in the ODE (17) leads to:

$$
\left[\left(k_{x}^{2}+k_{z p}^{2}\right) \mathbf{K}_{p}-\omega^{2} \mathbf{M}+i \omega \mathbf{E}\right]\left\{\begin{array}{l}
P^{s} \\
P^{f}
\end{array}\right\}=\left\{\begin{array}{l}
0 \\
0
\end{array}\right\},
$$

where $k_{x}^{2}+k_{z p}^{2}=k_{p}^{2}$, with $k_{p}$ the complex magnitude of the dilatational wave propagation vector $\mathbf{k}_{p}$. Non-trivial solutions for the eigenvector components $P^{\alpha}$ can be found if the determinant of the coefficient matrix of eqn (19) is zero. After some algebraic manipulations, the dispersion relation can be written alternatively as (Bowen and Reinicke, 1978; Garg et al., 1974) :

$$
\left[k_{p}^{2} C_{p 1}^{2}-\omega^{2}\right]\left[k_{p}^{2} C_{p 2}^{2}-\omega^{2}\right]+\frac{i \omega^{2}}{\chi}\left[k_{p}^{2} C_{p 0}^{2}-\omega^{2}\right]=0
$$

where the dimensionless frequency $\chi$ is defined as the ratio of the frequency $\omega$ to a characteristic frequency $\omega_{0}$, defined as $\omega_{0}=\operatorname{tr}\left(\mathbf{M}^{-1} \mathbf{E}\right)$, assuming that the viscosity correction factor $F=1 . \omega_{0}$ is inversely proportional to the permeability $k_{0}$. Biot (1956) and Smeulders (1992) use a slightly different characteristic frequency $\omega_{c}$, as introduced before. In eqn (20), $C_{p 0}$ is the P-wave velocity in the low frequency limit, while $C_{p 1}$ and $C_{p 2}$ are the $\mathrm{P}$-wave velocities in the non-dissipative porous solid. The dispersion relation (20) enables the calculation of the complex wavenumber $k_{p}$ in function of the frequency $\omega$. The dilatational dispersion relation is biquadratic, which reveals the existence of two P-waves (P1 and P2) in a saturated poroelastic medium (Biot, 1956). They will be referred to by a subscript $j=1,2$ in the following. The waves are dispersive and attenuated and involve a coupled motion between the solid skeleton and the pore fluid. The propagation velocities are defined as $C_{j p}=\omega / \operatorname{Re}\left(k_{j p}\right)$ and the attenuation coefficients as $q_{j p}=\operatorname{Im}\left(k_{j p}\right)$. The relative motion between both phases is described by means of complex frequency dependent coefficients $c o_{j p}$, so that $P_{j}^{f}=c o_{j p} P_{j}^{s}$, that follow immediately from eqns (19) as: 


$$
c o_{j p}=-\frac{\left(k_{j p}^{2} \alpha M-\omega^{2} \rho_{f}\right) n^{2}}{k_{j p}^{2} M n^{2}-\omega^{2} \rho_{f} a n+i \omega \xi} .
$$

Alternatively, modified coefficients $c o_{j p}^{\prime}=1+c o_{j p} / n$ that relate the absolute pore fluid displacement to the solid skeleton displacement will be used in the following.

The vertical wavenumbers $k_{z j p}$ of both dilatational waves can be calculated for each $k_{x}$ and $\omega$ from the relation $k_{x}^{2}+k_{z j p}^{2}=k_{j p}^{2}: k_{z j p}$ equals $\pm\left(k_{j p}^{2}-k_{x}^{2}\right)^{0.5}$ if $k_{x} \leqslant k_{j p}$, which corresponds to propagating waves in the $z$-direction. On the other hand, if $k_{x}>k_{j p}, k_{z j p}$ equals $\mp i\left(k_{x}^{2}-k_{j p}^{2}\right)^{0.5}$ and represents inhomogeneous waves with exponential decrease or increase in amplitude with $z$. Using the upper sign in both expressions for $k_{z j p}$, the solution (18) can be written more generally as:

$$
\widetilde{\Phi^{\alpha}}\left(k_{x}, z, \omega\right)=\sum_{j=1}^{2} P_{j}^{\alpha I} \exp \left(-i k_{z j p} z\right)+P_{j}^{\alpha R} \exp \left(+i k_{z j p} z\right),
$$

where the superscripts $I$ and $R$ refer to the incident (outgoing) waves, propagating in the positive $z$-direction, and the reflected (incoming) waves, propagating in the negative $z$ direction, respectively.

\subsection{Shear wave}

As the pore fluid does not sustain shear stress, the presence of a small amount of air bubbles in the pores of an unsaturated porous medium does not affect the characteristics of the shear (S) wave. Nevertheless, the following discussion recapitulates the terminology that will be needed further on.

The in-plane propagation of an S-wave in porous media is described by the second of the PDE (14). Application of a forward Fourier transformation on $t$ and $x$ transforms this PDE in the following ODE:

$$
\left[\left(k_{x}^{2}-\frac{\mathrm{d}^{2}}{\mathrm{~d} z^{2}}\right) \mathbf{K}_{s}-\omega^{2} \mathbf{M}+i \omega \mathbf{E}\right]\left\{\begin{array}{l}
\widetilde{\Psi^{s}} \\
\widetilde{\Psi^{f}}
\end{array}\right\}=\left\{\begin{array}{l}
0 \\
0
\end{array}\right\} .
$$

The following analytical solutions can be proposed for the wave potentials as a function of the independent coordinate $z$ :

$$
\widetilde{\Psi}^{\alpha}\left(k_{x}, z, \omega\right)=S^{\alpha} \exp \left(-i k_{z s} z\right) .
$$

$k_{z s}$ is the vertical component of the shear wave propagation vector $\mathbf{k}_{s}\left(k_{x}, k_{z s}\right)$. Introduction of the solutions (24) in the ODE (23) leads to:

$$
\left[\left(k_{x}^{2}+k_{z s}^{2}\right) \mathbf{K}_{s}-\omega^{2} \mathbf{M}+i \omega \mathbf{E}\right]\left\{\begin{array}{l}
S^{s} \\
S^{f}
\end{array}\right\}=\left\{\begin{array}{l}
0 \\
0
\end{array}\right\},
$$

where $k_{x}^{2}+k_{z s}^{2}=k_{s}^{2}$, with $k_{s}$ the complex magnitude of the shear wave propagation vector. Non-trivial solutions for the eigenvector components $S^{\alpha}$ can be found if the determinant of the coefficient matrix of eqn (25) is equal to zero. The dispersion relation can finally be written as :

$$
\left[k_{s}^{2} C_{s 1}^{2}-\omega^{2}\right]-\frac{i}{\chi}\left[k_{s}^{2} C_{s 0}^{2}-\omega^{2}\right]=0,
$$

where $C_{s 0}$ and $C_{s 1}$ are the shear wave velocities in the low frequency limit and in the nondissipative porous solid, respectively. The shear dispersion relation is bilinear and reveals the existence of a single shear wave in a saturated poroelastic medium (Biot, 1956), that is 
dispersive and attenuated and involves a coupled motion between the solid skeleton and the pore fluid. The wave propagation velocity is defined as $C_{s}=\omega / \operatorname{Re}\left(k_{s}\right)$ and the attenuation coefficient as $q_{s}=\operatorname{Im}\left(k_{s}\right)$. The relative motion between both phases is described by means of a complex frequency dependent coefficient $c o_{s}$, defined as $S^{f}=c o_{s} S^{s}$. This coefficient follows immediately from eqn (25) as :

$$
c o_{s}=-\frac{-\omega^{2} \rho_{f} n^{2}}{-\omega^{2} \rho_{f} a n+i \omega \xi} .
$$

Alternatively, a modified coefficient $c o_{s}^{\prime}=1+c o_{s} / n$ that relates the absolute pore fluid displacement to the solid skeleton displacement will be used in the following.

Following a similar argument as in the case of P-waves, the solution (24) can be written more generally as :

$$
\tilde{\Phi}^{\alpha}\left(k_{x}, z, \omega\right)=S^{\alpha I} \exp \left(-i k_{z s} z\right)+S^{\alpha R} \exp \left(+i k_{z s} z\right)
$$

\section{EXACT STIFFNESS FORMULATION}

\subsection{Variables in the wavenumber-frequency domain}

The motion of the pore fluid can be written in terms of the motion of the solid skeleton by means of the complex coefficients $c o_{j p}$ and $c o_{s}$. The eigenvector components related to the solid skeleton $\tilde{\mathbf{a}}=\left\{\begin{array}{llllll}S^{s I} & P_{1}^{s I} & P_{2}^{s I} & S^{s R} & P_{1}^{s R} & P_{2}^{s R}\end{array}\right\}^{T}$ then will be treated as the linearly independent set of variables. The vector $\tilde{\mathbf{a}}$ can be split into two subvectors $\tilde{\mathbf{a}}^{I}=\left\{\begin{array}{lll}S^{s I} & P_{1}^{s I} & P_{2}^{s I}\end{array}\right\}^{T}$ and $\tilde{\mathbf{a}}^{R}=\left\{\begin{array}{lll}S^{s R} & P_{1}^{s R} & P_{2}^{s R}\end{array}\right\}^{T}$ for the outgoing and incoming wave amplitudes, respectively.

The transformed displacements $\tilde{\mathbf{u}}=\left\{\begin{array}{llll}\tilde{u}_{x}^{s} & \tilde{u}_{z}^{s} & \tilde{w}_{x} & \tilde{w}_{z}\end{array}\right\}^{T}$ can be written in terms of the kernels $\tilde{\mathbf{a}}^{I}$ and $\tilde{\mathbf{a}}^{R}$ as :

$$
\tilde{\mathbf{u}}=\tilde{\mathbf{B}} \tilde{\mathbf{a}} \tilde{\mathbf{a}}=\left[\begin{array}{ll}
\tilde{\mathbf{B}}^{I} & \tilde{\mathbf{B}}^{R}
\end{array}\right]\left[\begin{array}{cc}
\tilde{\mathbf{Z}}^{I} & \mathbf{0} \\
\mathbf{0} & \tilde{\mathbf{Z}}^{R}
\end{array}\right]\left\{\begin{array}{c}
\tilde{\mathbf{a}}^{I} \\
\tilde{\mathbf{a}}^{R}
\end{array}\right\},
$$

where the diagonal submatrices $\tilde{\mathbf{Z}}^{I}$ and $\tilde{\mathbf{Z}}^{R}$ of the matrix $\tilde{\mathbf{Z}}$ describe the dependency of the solution on the vertical coordinate $z$ as follows :

$$
\begin{aligned}
\tilde{\mathbf{Z}}^{I} & =\operatorname{diag}\left\{\exp \left(-i k_{z s}\right) \exp \left(-i k_{z 1 p} z\right) \exp \left(-i k_{z 2 p} z\right)\right\} \\
\tilde{\mathbf{Z}}^{R} & =\operatorname{diag}\left\{\exp \left(+i k_{z s}\right) \exp \left(+i k_{z 1 p} z\right) \exp \left(+i k_{z 2 p} z\right)\right\} .
\end{aligned}
$$

The submatrices $\tilde{\mathbf{B}}^{I}$ and $\tilde{\mathbf{B}}^{R}$ of the matrix $\tilde{\mathbf{B}}$ depend on the excitation frequency $\omega$, the horizontal wavenumber $k_{x}$, the vertical wavenumbers $k_{z s}$ and $k_{z j p}$ and the coefficients $c o_{s}$ and $c o_{j p}$ as :

$$
\begin{gathered}
\tilde{\mathbf{B}}^{I}=\left[\begin{array}{ccc}
+i k_{z s} & -i k_{x} & -i k_{x} \\
-i k_{x} & -i k_{z 1 p} & -i k_{z 2 p} \\
+i k_{z s} c o_{s} & -i k_{x} c o_{1 p} & -i k_{x} c o_{2 p} \\
-i k_{x} c o_{s} & -i k_{z p} c o_{1 p} & -i k_{z 2 p} c o_{2 p}
\end{array}\right], \\
\tilde{\mathbf{B}}^{R}=\left[\begin{array}{ccc}
-i k_{z s} & -i k_{x} & -i k_{x} \\
-i k_{x} & +i k_{z 1 p} & +i k_{z 2 p} \\
-i k_{z s} c o_{s} & -i k_{x} c o_{1 p} & -i k_{x} c o_{2 p} \\
-i k_{x} c o_{s} & +i k_{z 1 p} c o_{1 p} & +i k_{z 2 p} c o_{2 p}
\end{array}\right] .
\end{gathered}
$$

The four displacement components in the vector $\tilde{\mathbf{u}}$ are linearly dependent on the kernels $\tilde{\mathbf{a}}^{I}$ 
or $\tilde{\mathbf{a}}^{R}$. Thus, a reduced set of linearly independent transformed displacements $\tilde{\mathbf{u}}_{r}=\left\{\begin{array}{lll}\tilde{u}_{x}^{s} & i \tilde{u}_{z}^{s} & i \tilde{w}_{z}\end{array}\right\}^{T}$ will be used in the following:

$$
\tilde{\mathbf{u}}_{r}=\left[\begin{array}{llll}
1 & 0 & 0 & 0 \\
0 & i & 0 & 0 \\
0 & 0 & 0 & i
\end{array}\right] \tilde{\mathbf{u}}=\mathbf{T}_{u} \tilde{\mathbf{u}}=\mathbf{T}_{u} \tilde{\mathbf{B}} \tilde{\mathbf{Z}} \tilde{\mathbf{a}}=\tilde{\mathbf{D}} \tilde{\mathbf{Z}} \tilde{\mathbf{a}}
$$

The transformation matrix $\mathbf{T}_{u}$ selects the appropriate elements from the transformed displacement vector $\tilde{\mathbf{u}}$; in order to obtain symmetric coefficient matrices in the following derivations, the vertical displacement components are multiplied by the imaginary unit $i$. The latter is not necessary when Fourier cosine and sine or Hankel transformations are used. Submatrices of $\tilde{\mathbf{D}}=\mathbf{T}_{u} \tilde{\mathbf{B}}$ are defined as $\tilde{\mathbf{D}}^{I}=\mathbf{T}_{u} \tilde{\mathbf{B}}^{I}$ and $\tilde{\mathbf{D}}^{R}=\mathbf{T}_{u} \tilde{\mathbf{B}}^{R}$.

The two-dimensional transformed strains $\tilde{\boldsymbol{\varepsilon}}=\left\{\begin{array}{llll}\widetilde{\varepsilon}_{x x}^{s} & \widetilde{\varepsilon}_{z z}^{s} & \tilde{\gamma}_{x z}^{s} & \tilde{\zeta}^{u}\end{array}\right\}^{T}$, with $\tilde{\gamma}_{x z}^{s}$ the "engineering shear strain", are equal to :

$$
\tilde{\boldsymbol{\varepsilon}}=\tilde{\mathbf{C}} \tilde{\mathbf{Z}} \tilde{\mathbf{a}}=\left[\begin{array}{ll}
\tilde{\mathbf{C}}^{I} & \tilde{\mathbf{C}}^{R}
\end{array}\right]\left[\begin{array}{cc}
\tilde{\mathbf{Z}}^{I} & \mathbf{0} \\
\mathbf{0} & \tilde{\mathbf{Z}}^{R}
\end{array}\right]\left\{\begin{array}{c}
\tilde{\mathbf{a}}^{I} \\
\tilde{\mathbf{a}}^{R}
\end{array}\right\}
$$

Here, the submatrices $\tilde{\mathbf{C}}^{I}$ and $\tilde{\mathbf{C}}^{R}$ of the matrix $\tilde{\mathbf{C}}$ are :

$$
\begin{gathered}
\tilde{\mathbf{C}}^{I}=\left[\begin{array}{ccc}
+k_{x} k_{z s} & -k_{x}^{2} & -k_{x}^{2} \\
-k_{x} k_{z s} & -k_{z 1 p}^{2} & -k_{z 2 p}^{2} \\
-k_{x}^{2}+k_{z s}^{2} & -2 k_{x} k_{z 1 p} & -2 k_{x} k_{z 2 p} \\
0 & -k_{1 p}^{2} c o_{1 p} & -k_{2 p}^{2} c o_{2 p}
\end{array}\right], \\
\tilde{\mathbf{C}}^{R}=\left[\begin{array}{ccc}
-k_{x} k_{z s} & -k_{x}^{2} & -k_{x}^{2} \\
+k_{x} k_{z s} & -k_{z 1 p}^{2} & -k_{z 2 p}^{2} \\
-k_{x}^{2}+k_{z s}^{2} & +2 k_{x} k_{z 1 p} & +2 k_{x} k_{z 2 p} \\
0 & -k_{1 p}^{2} c o_{1 p} & -k_{2 p}^{2} c o_{2 p}
\end{array}\right] .
\end{gathered}
$$

The transformed modified strain vector $\tilde{\boldsymbol{\varepsilon}}_{r}=\left\{\begin{array}{llll}i \widetilde{e}^{s} & i \widetilde{\varepsilon}_{z z}^{s} & \tilde{\gamma}_{x z}^{s} & i \tilde{\zeta}\end{array}\right\}^{T}$ is introduced as :

$$
\tilde{\boldsymbol{\varepsilon}}_{r}=\left[\begin{array}{cccc}
i & i & 0 & 0 \\
0 & i & 0 & 0 \\
0 & 0 & 1 & 0 \\
0 & 0 & 0 & i
\end{array}\right] \tilde{\boldsymbol{\varepsilon}}=\mathbf{T}_{\varepsilon} \tilde{\boldsymbol{\varepsilon}}=\mathbf{T}_{\varepsilon} \tilde{\mathbf{C}} \tilde{\mathbf{Z}} \tilde{\mathbf{a}}=\tilde{\mathbf{E}} \tilde{\mathbf{Z}} \tilde{\mathbf{a}}
$$

The transformation matrix $\mathbf{T}_{\varepsilon}$ replaces the horizontal strain $\widetilde{\varepsilon}_{x x}^{s}$ in the solid skeleton by the volumetric strain $\tilde{e}^{s}$ and multiplies some variables with the imaginary unit. Submatrices of $\tilde{\mathbf{E}}=\mathbf{T}_{\varepsilon} \tilde{\mathbf{C}}$ are defined as $\tilde{\mathbf{E}}^{I}=\mathbf{T}_{\varepsilon} \tilde{\mathbf{C}}^{I}$ and $\tilde{\mathbf{E}}^{R}=\mathbf{T}_{\varepsilon} \tilde{\mathbf{C}}^{R}$.

The transformed stresses $\tilde{\boldsymbol{\sigma}}=\left\{\begin{array}{llll}\tilde{\sigma}_{x x} & \tilde{\sigma}_{z z} & \tilde{\sigma}_{x z} & -\tilde{p}\end{array}\right\}^{T}$ are related to the transformed strains $\tilde{\boldsymbol{\sigma}}$ by:

$$
\tilde{\boldsymbol{\sigma}}=\mathbf{K} \tilde{\boldsymbol{\varepsilon}}
$$

The matrix $\mathbf{K}$ of poroelastic coefficients reads : 


$$
\mathbf{K}=\left[\begin{array}{cccc}
\lambda^{s}+2 \mu^{s}+\alpha^{2} M & \lambda^{s}+\alpha^{2} M & 0 & \alpha M \\
\lambda^{s}+\alpha^{2} M & \lambda^{s}+2 \mu^{s}+\alpha^{2} M & 0 & \alpha M \\
0 & 0 & \mu^{s} & 0 \\
\alpha M & \alpha M & 0 & M
\end{array}\right] .
$$

Since the pore fluid cannot sustain any shear stress, only three stress components have to be specified at any boundary perpendicular to the $z$-axis. Therefore, the transformed reduced stress vector $\tilde{\boldsymbol{\sigma}}_{r}=\left\{\begin{array}{lll}\tilde{\sigma}_{x z} & i \tilde{\sigma}_{z z} & -i \tilde{p}\end{array}\right\}^{T}$ is defined as:

$$
\tilde{\boldsymbol{\sigma}}_{r}=\left[\begin{array}{llll}
0 & 0 & 1 & 0 \\
0 & i & 0 & 0 \\
0 & 0 & 0 & i
\end{array}\right] \tilde{\boldsymbol{\sigma}}=\mathbf{T}_{\sigma} \tilde{\boldsymbol{\sigma}}
$$

where the transformation matrix $\mathbf{T}_{\sigma}$ selects the appropriate elements from the transformed stress vector $\tilde{\boldsymbol{\sigma}}$ and multiplies some variables with the imaginary unit. It is easy to demonstrate that the transformed reduced stresses $\tilde{\boldsymbol{\sigma}}_{r}$ are related to the transformed modified strains $\tilde{\boldsymbol{\varepsilon}}_{r}$ by a reduced matrix of constitutive coefficients $\mathbf{K}_{r}=\mathbf{T}_{\sigma} \mathbf{K} \mathbf{T}_{\varepsilon}^{-1}$.

\subsection{Saturated layer element}

For a saturated layer element the transformed variables are expressed in terms of the incident as well as the reflected kernels. The displacements $\mathbf{u}^{e}$ at both interfaces of a layer element (Fig. 1) are:

$$
\tilde{\mathbf{u}}^{e}=\left\{\begin{array}{l}
\tilde{\mathbf{u}}_{1}^{e} \\
\tilde{\mathbf{u}}_{2}^{e}
\end{array}\right\}=\left\{\begin{array}{l}
\tilde{\mathbf{u}}_{r}(z=0) \\
\tilde{\mathbf{u}}_{r}(z=L)
\end{array}\right\}=\left[\begin{array}{ll}
\tilde{\mathbf{D}}^{I} \tilde{\mathbf{Z}}^{I}(z=0) & \tilde{\mathbf{D}}^{R} \tilde{\mathbf{Z}}^{R}(z=0) \\
\tilde{\mathbf{D}}^{I} \tilde{\mathbf{Z}}^{I}(z=L) & \tilde{\mathbf{D}}^{R} \tilde{\mathbf{Z}}^{R}(z=L)
\end{array}\right]\left\{\begin{array}{c}
\tilde{\mathbf{a}}^{I} \\
\tilde{\mathbf{a}}^{R}
\end{array}\right\},
$$

where the submatrices $\tilde{\mathbf{Z}}^{I}(z=0)$ and $\tilde{\mathbf{Z}}^{R}(z=0)$ reduce to the $3 \times 3$ identity matrix.

The element tractions $\tilde{\mathbf{T}}^{e}$ at both interfaces of a layer element can be written in terms of the transformed reduced stresses by application of Cauchy's stress principle at the horizontal interfaces $z=0$ and $z=L$. There, the unit outward normal vector is directed in the negative and positive $z$-directions, respectively. Using the relations introduced in the previous subsection, the tractions at both interfaces of the layer element are:

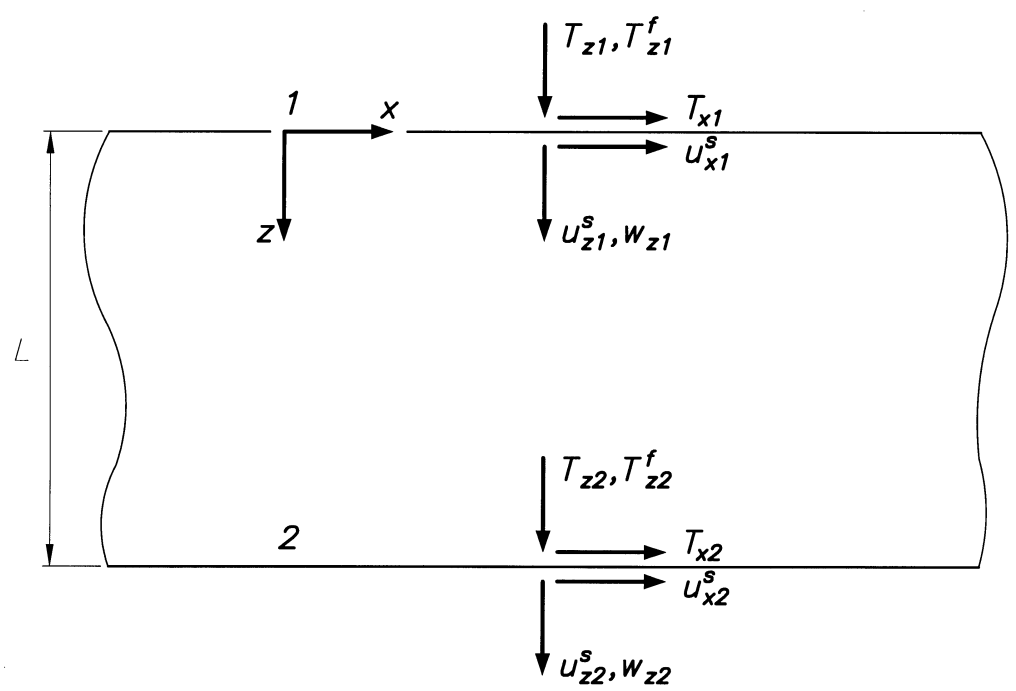

Fig. 1. Saturated layer element. 


$$
\begin{aligned}
\tilde{\mathbf{T}}^{e} & =\left\{\begin{array}{l}
\tilde{\mathbf{T}}_{1}^{e} \\
\tilde{\mathbf{T}}_{2}^{e}
\end{array}\right\}=\left\{\begin{array}{l}
-\tilde{\boldsymbol{\sigma}}_{r}(z=0) \\
+\tilde{\boldsymbol{\sigma}}_{r}(z=L)
\end{array}\right\} \\
& =\left[\begin{array}{ll}
-\mathbf{K}_{r} \tilde{\mathbf{E}}^{I} \tilde{\mathbf{Z}}^{I}(z=0) & -\mathbf{K}_{r} \tilde{\mathbf{E}}^{R} \tilde{\mathbf{Z}}^{R}(z=0) \\
+\mathbf{K}_{r} \tilde{\mathbf{E}}^{I} \tilde{\mathbf{Z}}^{I}(z=L) & +\mathbf{K}_{r} \tilde{\mathbf{E}}^{R} \mathbf{Z}^{R}(z=L)
\end{array}\right]\left\{\begin{array}{l}
\tilde{\mathbf{a}}^{I} \\
\tilde{\mathbf{a}}^{R}
\end{array}\right\} .
\end{aligned}
$$

Elimination of the wave amplitudes from eqns (39) and (40) allows the element displacements $\tilde{\mathbf{u}}^{e}$ to be related to the element tractions $\tilde{\mathbf{T}}^{e}$ as :

$$
\tilde{\mathbf{K}}^{e} \tilde{\mathbf{u}}^{e}=\tilde{\mathbf{T}}^{e}
$$

or, in blockwise notation:

$$
\left[\begin{array}{ll}
\tilde{\mathbf{K}}_{11}^{e} & \tilde{\mathbf{K}}_{12}^{e} \\
\tilde{\mathbf{K}}_{21}^{e} & \tilde{\mathbf{K}}_{22}^{e}
\end{array}\right]\left\{\begin{array}{c}
\tilde{\mathbf{u}}_{1}^{e} \\
\tilde{\mathbf{u}}_{2}^{e}
\end{array}\right\}=\left\{\begin{array}{l}
\tilde{\mathbf{T}}_{1}^{e} \\
\tilde{\mathbf{T}}_{2}^{e}
\end{array}\right\}
$$

The matrix $\tilde{\mathbf{K}}^{e}$ is the $6 \times 6$ complex stiffness matrix of the saturated layer element. As $\tilde{\mathbf{K}}^{e}$ is symmetric, $\tilde{\mathbf{K}}_{21}^{e}=\tilde{\mathbf{K}}_{12}^{e T}$. Moreover, a symmetry argument can be used to demonstrate that the elements of the submatrix $\widetilde{\mathbf{K}}_{22}^{e}$ follow immediately from the elements of $\widetilde{\mathbf{K}}_{11}^{e}$. As a result, only the elements of the submatrices $\tilde{\mathbf{K}}_{11}^{e}$ and $\tilde{\mathbf{K}}_{12}^{e}$ have to be determined explicitly.

It is not advisable to calculate the layer stiffness matrix as the product of the coefficient matrix in eqn (40) and the inverse of the coefficient matrix in eqn (39). Due to the presence of the submatrices $\tilde{\mathbf{Z}}^{I}(z=L)$ and $\tilde{Z}^{R}(z=L)$, the latter is severely ill-conditioned for large values of $k_{x} L$, which would result in very poor computational results. Instead, by matrix algebra it is easy to obtain the following expressions for the submatrices $\tilde{\mathbf{K}}_{11}^{e}$ and $\tilde{\mathbf{K}}_{12}^{e}$ in terms of submatrices introduced earlier:

$$
\begin{aligned}
& \tilde{\mathbf{K}}_{11}^{e}=-\left[\mathbf{K}_{r} \tilde{\mathbf{E}}^{I}-\mathbf{K}_{r} \tilde{\mathbf{E}}^{R} \tilde{\mathbf{Z}}^{I} \tilde{\mathbf{D}}^{R-1} \tilde{\mathbf{D}}^{I} \tilde{\mathbf{Z}}^{I}\right]\left[\tilde{\mathbf{D}}^{I}-\tilde{\mathbf{D}}^{R} \tilde{\mathbf{Z}}^{I} \tilde{\mathbf{D}}^{R-1} \tilde{\mathbf{D}}^{I} \tilde{\mathbf{Z}}^{I}\right]^{-1}, \\
& \tilde{\mathbf{K}}_{12}^{e}=\left[\mathbf{K}_{r} \tilde{\mathbf{E}}^{I}-\mathbf{K}_{r} \tilde{\mathbf{E}}^{R} \tilde{\mathbf{D}}^{R-1} \tilde{\mathbf{D}}^{I}\right] \tilde{\mathbf{Z}}^{I}\left[\tilde{\mathbf{D}}^{I}-\tilde{\mathbf{D}}^{R} \tilde{\mathbf{Z}}^{I} \tilde{\mathbf{D}}^{R-1} \tilde{\mathbf{D}}^{I} \tilde{\mathbf{Z}}^{I}\right]^{-1}
\end{aligned}
$$

Here, it is understood that the submatrix $\tilde{\mathbf{Z}}^{I}$ is evaluated at $z=L$. For $k_{x}=0$, corresponding to the one-dimensional case, the horizontal and vertical motions or the contributions to the shear and dilatational motion decouple. Expressions (43) are stable for large values of $k_{x}$. The submatrix $\tilde{\mathbf{K}}_{11}^{e}$ tends to $-\mathbf{K}_{r} \tilde{\mathbf{E}}^{I} \tilde{\mathbf{D}}^{I-1}$, the expression of the element stiffness matrix of the halfspace, as will be derived in the next subsection. Furthermore, the submatrix $\tilde{\mathbf{K}}_{12}^{e}$ tends to zero for large $k_{x}$, since $\tilde{\mathbf{Z}}^{I}$ tends to zero. This confirms that, as $k_{x}$ increases, the uncoupling of the modes shifts towards an uncoupling of the interfaces.

Although the expressions (43) allow drawing some conclusions for limiting values of $k_{x}$, their straightforward numerical evaluation is still not advisable, as some of the submatrices are singular for zero frequency (static case) and/or horizontal (one-dimensional case) and vertical wavenumber. In view of numerical accuracy and computational efficiency, it is therefore very useful to derive analytical expressions for the elements of the layer stiffness matrix, as well as limiting expressions for zero $\omega$ and $k_{x}$. Whereas analytical expressions and corresponding limiting expressions for the elements of the dry layer element stiffness matrix are available since two decades (Kausel and Roesset, 1981), the complexity of the equations has compromised the derivation of the corresponding expressions in the (un)saturated case. Only very recently, Rajapakse and Senjuntichai (1995) have been successful in deriving analytical expressions for the elements of $\tilde{\mathbf{K}}^{e}$ through symbolic manipulation with Mathematica (Wolfram, 1991). However, these authors did not derive limiting expressions for vanishing $\omega$ and/or $k_{x}$ which are recommended for a stable numerical implementation. 


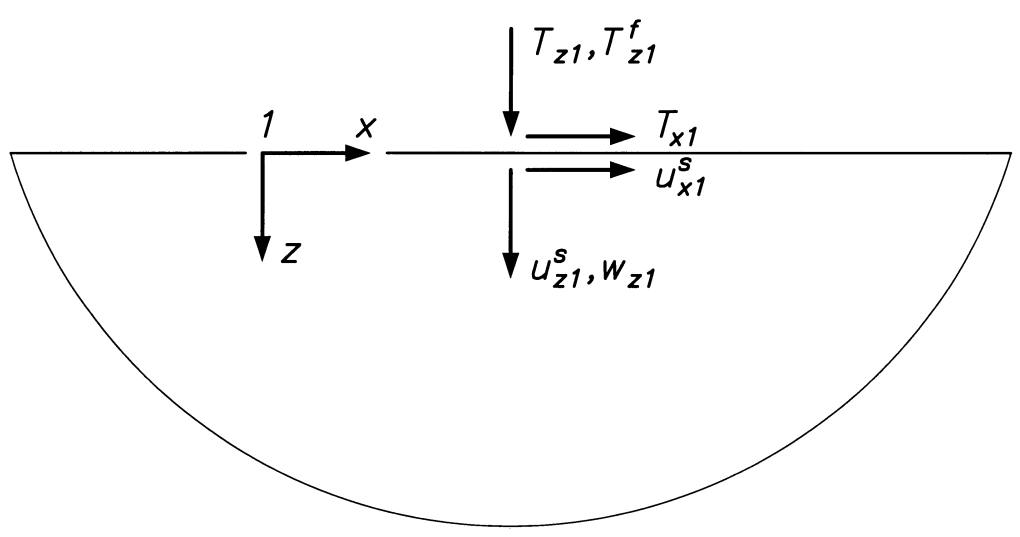

Fig. 2. Saturated halfspace element.

\subsection{Saturated halfspace element}

The saturated halfspace element models the propagation of waves in a semi-infinite halfspace. The amplitudes of the harmonic waves should be non-increasing functions of the distance travelled in the direction of wave propagation. Therefore, only the outgoing waves will be considered. The displacements $\tilde{\mathbf{u}}^{e}$ at the single interface of a halfspace element can be calculated by evaluation of eqn (32) at $z=0$ as:

$$
\tilde{\mathbf{u}}^{e}=\tilde{\mathbf{u}}_{r}(z=0)=\tilde{\mathbf{D}}^{I} \tilde{\mathbf{Z}}^{I}(z=0) \tilde{\mathbf{a}}^{I} .
$$

The element traction $\tilde{\mathbf{T}}^{e}$ can be written in terms of the transformed reduced stresses by application of Cauchy's stress principle on the horizontal interface $z=0$ with an outward unit normal vector in the direction of the negative $z$-axis. Using the relations introduced previously, the tractions at the single interface of the halfspace element are:

$$
\tilde{\mathbf{T}}^{e}=-\tilde{\boldsymbol{\sigma}}_{r}(z=0)=-\mathbf{K}_{r} \tilde{\mathbf{E}}^{I} \tilde{\mathbf{Z}}^{I}(z=0) \tilde{\mathbf{a}}^{I} .
$$

In both eqns (44) and (45), the matrix $\tilde{\mathbf{Z}}^{I}(z=0)$ is equal to the $3 \times 3$ identity matrix. The element tractions $\tilde{\mathbf{T}}^{e}$ are related to the element displacements $\tilde{\mathbf{u}}^{e}$ after elimination of the outgoing wave amplitudes $\tilde{\mathbf{a}}^{I}$ from eqns (44) and (45) :

$$
\tilde{\mathbf{K}}^{e} \tilde{\mathbf{u}}^{e}=\tilde{\mathbf{T}}^{e}
$$

where the $3 \times 3$ complex symmetrical element stiffness matrix $\widetilde{\mathbf{K}}^{e}$ of the saturated halfspace element is equal to :

$$
\tilde{\mathbf{K}}^{e}=-\mathbf{K}_{r} \tilde{\mathbf{E}}^{I} \tilde{\mathbf{D}}^{I-1} .
$$

Analytical expressions for the elements of the stiffness matrix $\tilde{\mathbf{K}}^{e}$ can be derived through symbolic manipulation of expression (47) with Mathematica (Wolfram, 1991). Limiting expressions for vanishing frequency (static case) and/or horizontal wavenumber (onedimensional case) are readily available.

\subsection{Assembly of equations}

For each frequency $\omega$ and horizontal wavenumber $k_{x}$, the element stiffness matrices $\tilde{\mathbf{K}}^{e}$ are calculated as explained above and assembled in a global stiffness matrix $\tilde{\mathbf{K}}^{S}$ relating the tractions $\tilde{\mathbf{T}}^{S}$ and the displacements $\tilde{\mathbf{u}}^{S}$ : 


$$
\tilde{\mathbf{K}}^{S} \tilde{\mathbf{u}}^{S}=\tilde{\mathbf{T}}^{S}
$$

The assembly process establishes the equilibrium at each horizontal interface, assuming the continuity in pairs of $\sigma_{x z}$ and $u_{x}^{s}, \sigma_{z z}$ and $u_{z}^{s}$, and $p$ and $w_{z}$ (Bourbié et al., 1987; Deresiewicz and Skalak, 1963). The complex stiffness matrix $\tilde{\mathbf{K}}^{S}$ is symmetrical and banded. Since the frequency and wavenumber dependent eigenvectors are used as shape functions for the formulation of the element stiffness matrices $\tilde{\mathbf{K}}^{e}$, the mass distribution is treated exactly without the need of subdividing a member into smaller elements. Wave propagation within an element is treated exactly and elements can extend from one interface to another.

This gives a substantial reduction in the size of the system represented by eqn (48) compared as to a thin layer formulation (Bougacha et al., 1993). In this method, for the discretization in the vertical direction, polynomial shape functions are used instead of solutions of the wave equations. Consequently, the layer thickness should be small enough with respect to the smallest wavelength in the response. Moreover, the thin layer method was originally formulated for a layered stratum on rigid bedrock. Wave propagation in a semi-infinite layered halfspace could be treated as a hybrid formulation, by combining a thin layer formulation for the layers with an exact halfspace element, as derived in the foregoing subsection.

\subsection{Possible applications}

4.5.1. Free-field response. In a first application, the free-field response of a multilayered medium due to an incident plane wave (a P1, P2 or SV wave) with an angle of incidence $\varphi$ at a frequency $\omega$ is considered. This procedure can be used to calculate the free field response of a site due to an incident seismic wave in a dynamic soil-structure interaction problem. Another possible application is the determination of the acoustical impedance of isotropic multilayered porous materials.

When an incident wave impinges on a layered medium, the displacements $\tilde{\mathbf{u}}^{e}$ of the interface with the underlying halfspace not only depend on the outgoing wave amplitudes $\tilde{\mathbf{a}}^{I}$, but also on the imposed incident waves $\tilde{\mathbf{a}}^{R}=\tilde{\mathbf{a}}^{\text {inc }}$ as :

$$
\tilde{\mathbf{u}}^{e}=\tilde{\mathbf{u}}_{r}(z=0)=\tilde{\mathbf{D}}^{I} \tilde{\mathbf{a}}^{I}+\tilde{\mathbf{D}}^{R} \tilde{\mathbf{a}}^{\mathrm{inc}} .
$$

It is understood here that $\tilde{\mathbf{Z}}^{I}(z=0)$ and $\tilde{\mathbf{Z}}^{R}(z=0)$ are equal to the $3 \times 3$ unit matrix. Similarly, the element tractions can be written as :

$$
\tilde{\mathbf{T}}^{e}=-\tilde{\boldsymbol{\sigma}}_{r}(z=0)=-\mathbf{K}_{r} \tilde{\mathbf{E}}^{I} \tilde{\mathbf{a}}^{I}-\mathbf{K}_{r} \tilde{\mathbf{E}}^{R} \tilde{\mathbf{a}}^{\text {inc }}
$$

Elimination of the unknown $\tilde{\mathbf{a}}^{I}$ from eqns (49) and (50) gives rise to the following element equilibrium equation of the halfspace element :

$$
\tilde{\mathbf{K}}^{e} \tilde{\mathbf{u}}^{e}=\tilde{\mathbf{T}}^{e}+\left(\mathbf{K}_{r} \tilde{\mathbf{E}}^{R}-\mathbf{K}_{r} \tilde{\mathbf{E}}^{I} \tilde{\mathbf{D}}^{I-1} \tilde{\mathbf{D}}^{R}\right) \tilde{\mathbf{a}}^{\mathrm{inc}}
$$

where the second term on the right hand side accounts for the incident wave. This equation is assembled in the global equilibrium eqn (48). A unit vector $\tilde{\mathbf{a}}^{\text {inc }}$ represents an incident wave of a particular type and unit amplitude. A specific choice of the angle of incidence $\varphi$ and the excitation frequency $\omega$ determines the (complex) horizontal wavenumber, resulting in a one-dimensional amplification problem to be solved.

4.5.2. Surface waves. A second application involves the calculation of the natural modes of vibration or free surface waves in a multilayered dry, saturated or unsaturated halfspace. The natural modes of vibration are equal to the displacements $\tilde{\mathbf{u}}^{S}$ when the load vector $\tilde{\mathbf{T}}^{S}$ equals zero in eqn (48). Non-trivial solutions for $\tilde{\mathbf{u}}^{S}$ can be obtained if the coefficient matrix $\tilde{\mathbf{K}}^{S}$ is singular or if the determinant of $\tilde{\mathbf{K}}^{S}$ is equal to zero: 


$$
\operatorname{det} \widetilde{\mathbf{K}}^{S}=0 \text {. }
$$

This equation corresponds to a transcendental eigenvalue problem in terms of the real frequency $\omega$ and the complex horizontal wavenumber $k_{x}$, which imaginary part represents wave attenuation in the horizontal direction. This eigenvalue problem has an infinite number of solutions and must be solved by search techniques. We have used Powell's hybrid method (Draelants, 1994), based on an algorithm that combines the advantages of the Levenberg-Marquardt approach and update techniques, improving the convergence of the search algorithm and reducing the computational cost.

The present formulation can be considered as a generalization of more classical work where surface waves in a dry halfspace (Rayleigh, 1887), a dry layer on a halfspace (Achenbach and Epstein, 1967), a dry multilayered halfspace (Haskell, 1953), a saturated halfspace (Deresiewicz, 1962; Jones, 1961) and a "partially" saturated halfspace (Philippacopoulos, 1987) have been considered.

The advantage of using, alternatively, a thin layer method is that a quadratic rather than a transcendental eigenvalue problem is obtained. The method is restricted however to the calculation of normal wave modes in a layered stratum on a rigid bedrock (Bougacha et al., 1993). If a hybrid formulation is used where thin layers are combined with a halfspace element, the eigenvalue problem is transcendental again.

4.5.3. Forced vibrations. A third application involves the calculation of the response of a layered halfspace to an external transient loading. At each interface between layers, the displacements or tractions can be prescribed. The traction can be represented by a function $T(x, t)=S(x) F(t)$ where $S(x)$ and $F(t)$ denote the spatial and temporal variation of the loading, respectively.

The function $F(t)$ is transformed to the frequency domain by means of a Fast Fourier Transform (FFT) algorithm. The spatial distribution of the loading $S(x)$ is limited to some special functions (e.g. Dirac impulse, uniform distribution, normal distribution) of which the spectral content in the horizontal wavenumber domain can be evaluated analytically (Bateman, 1954).

The inverse integral transformations from the wavenumber to the spatial domain are of the general form $\int_{0}^{\infty} f\left(k_{x}, z, \omega\right) \cos \left(k_{x} x\right) \mathrm{d} k_{x}$ or $\int_{0}^{\infty} f\left(k_{x}, z, \omega\right) \sin \left(k_{x} x\right) \mathrm{d} k_{x}$ for the in-plane case and $\int_{0}^{\infty} f\left(k_{r}, z, \omega\right) k_{r} J_{n}\left(k_{r} r\right) \mathrm{d} k_{r}$ for the axisymmetric case, with $n$ the order of the Bessel function. The following characteristics of the integrand are important:

-The function $f\left(k_{x}, z, \omega\right)$ follows from the solution of the system of eqns (48). Although the relative increase in computation time due to an increase in the number of layers is not particularly large ( $\mathrm{Xu}$ and Mal, 1987), its evaluation is expensive.

-The function $f\left(k_{x}, z, \omega\right)$ exhibits dense oscillations, which are due to exponential terms in the diagonal submatrices $\tilde{\mathbf{Z}}^{I}$ and $\tilde{\mathbf{Z}}^{R}$ for particular values of $k_{x}$. The presence of very low speed layers is likely to cause rapid oscillations in some parts of the integrand, which are irregular as their exact location and nature cannot be predicted ( $\mathrm{Xu}$ and $\mathrm{Mal}, 1987$ ).

-In the absence of material dissipation, the function $f\left(k_{x}, z, \omega\right)$ becomes zero for certain values of $k_{x}$, which correspond to the surface wave poles of the integrand. The number of poles is almost proportional to the frequency and the total thickness of the layers above the halfspace. Several techniques to remove the poles from the path of integration have been reported in literature (Apsel and Luco, 1983; Bouchon and Aki, 1977; Kundu and Mal, 1985; Luco and Apsel, 1983). We have added material damping as this method of pole removal corresponds to a physical reality.

- The kernel functions in the integral transforms introduce rapid oscillations for large values of the horizontal source-receiver distance.

In view of the foregoing remarks, an efficient quadrature scheme is needed for the evaluation of the inverse wavenumber integrals. According to $\mathrm{Xu}$ and Mal (1985) an adaptive algorithm with self-adjusting interval, concentrating abscissas around regions of sharp variations in $f\left(k_{x}, z, \omega\right)$ and taking full advantage of previously computed values of the integrand, is most useful in order to obtain an accurate evaluation of the wavenumber integral with a minimum number of function evaluations. 
Different quadrature formula can be obtained depending on the method used to estimate the derivatives of the expanded function. Apsel and Luco (1983) have used Lagrange's five point formula, representing the integrand locally with a quartic polynomial for variable step size. They use a hybrid quadrature scheme, in a sense that, depending on the magnitude of the arguments of the Bessel functions, a non-Filon method or a Filon method is used. We have followed Fraser and Gettrust (1984) and used a generalized Filon method which makes use of an asymptotic expansion in a way that does not depend on the accuracy of the expansion (Van den Broeck et al., 1993). Alternative quadrature schemes have been investigated by Kundu and Mal (1985) and Xu and Mal (1987) but have not been used in the present implementation.

The inverse transformation from the frequency to the time is performed by an inverse FFT algorithm.

\section{NUMERICAL EXAMPLE}

In the following numerical example, we will study the effect of a moving water table on the propagation of transient waves in an isotropic axisymmetric halfspace. Furthermore, the influence of a small amount of air bubbles in the pores of the saturated medium will be illustrated. The halfspace consists of sand of Mol, whose dynamic material characteristics are summarized in a first subsection. The dispersion and attenuation characteristics of the dilatational and shear waves are illustrated next. Subsequently, wave propagation in an axisymmetric layered halfspace is studied in the wavenumber-frequency domain as well as the space-time domain.

\subsection{Material characteristics}

The (un)saturated material under consideration is a sand of Mol, composed of subangular quartz particles with a mean grain diameter $d_{50}=0.195 \mathrm{~mm}$. The connected pore space is saturated with water. The physical characteristics of sand of Mol have been summarized by Van Impe (1981), while its dynamic material characteristics have been studied at low (Yoon, 1992) and high (Van Impe, 1981; Thooft, 1992) deformation ratios. The porosity $n$ equals 0.388 . The sand grain density equals $\rho_{s}=2650 \mathrm{~kg} / \mathrm{m}^{3}$ and the pore fluid density is equal to $\rho_{f}=1000 \mathrm{~kg} / \mathrm{m}^{3}$. The resulting mixture density equals $\rho=2009.8$ $\mathrm{kg} / \mathrm{m}^{3}$.

The solid skeleton has a Young modulus $E^{s}=2.983 \times 10^{8} \mathrm{~N} / \mathrm{m}^{2}$ and a Poisson coefficient $v^{s}=1 / 3$. The corresponding Lamé coefficients are $\lambda^{s}=2.236 \times 10^{8} \mathrm{~N} / \mathrm{m}^{2}$ and $\mu^{s}=1.1186 \times 10^{8} \mathrm{~N} / \mathrm{m}^{2}$. The bulk modulus of the pore fluid is $K_{l}=2.2 \times 10^{9} \mathrm{~N} / \mathrm{m}^{2}$. According to the classical soil mechanics' assumption, the Biot coefficient $\alpha=1.0$.

In the (un)saturated case, the Darcy permeability is taken to be $k=1.0 \times 10^{-4} \mathrm{~m} / \mathrm{s}$. Accounting for the dynamic viscosity $\eta_{d}=1.002 \times 10^{-3} \mathrm{Ns} / \mathrm{m}^{2}$ and the density of the pore fluid, the specific permeability $k_{I}=\eta_{d} k / \gamma_{w}=10.214 \times 10^{-12} \mathrm{~m}^{2}$. Due to the lack of experimental data, we have taken the tortuosity factor $a=1.789$, in agreement with Berryman's relation $a=(1+n) / 2 n$ (Bourbié et al., 1987). Moreover, a frequency-dependent correction factor $F(\omega)$ is used to account for the dynamic permeability, following Johnson et al. (1987).

In the unsaturated case, the presence of $0.1 \%$ of air bubbles with radius $R_{0}=0.5 \mathrm{~mm}$ will be assumed. The density of the air is $\rho_{g}=1.205 \mathrm{~kg} / \mathrm{m}^{3}$, the gas bubble pressure in the equilibrium state is $p_{g 0}=1.0 \times 10^{5} \mathrm{~N} / \mathrm{m}^{2}$, the thermal diffusivity of the gas phase is $a_{g}=1.87 \times 10^{-5} \mathrm{~m}^{2} / \mathrm{s}$, the specific heat ratio is $\gamma=1.4$ and the surface tension is $\sigma=0.07$ $\mathrm{N} / \mathrm{m}$.

\subsection{Dispersion relations for isotropic materials}

5.2.1. Dry poroelastic material. In the dry poroelastic material, the dilatational and shear wave are non-dispersive and unattenuated in the absence of material damping. The wave propagation velocities are equal to $C_{p}=\sqrt{\left(\lambda^{s}+2 \mu^{s}\right) / \rho^{s}}=525.2 \mathrm{~m} / \mathrm{s}$ and $C_{s}=\sqrt{\mu^{s} / \rho^{s}}=262.6 \mathrm{~m} / \mathrm{s}$, respectively. The ratio of $C_{s}$ and $C_{p}$ will be denoted as $s$. This 
ratio is equal to $\sqrt{\left(1-2 v^{s}\right) /\left(2-2 v^{s}\right)}$ and only depends on Poisson's ratio $v^{s}$. As $v^{s}$ is equal to $1 / 3, s$ is equal to 0.5 .

5.2.2. Saturated poroelastic material. In this example, the characteristic (angular) frequency $\omega_{0}$, governing the transition from low to high frequency behaviour, is equal to $23,850 \mathrm{rad} / \mathrm{s}$. Figure 3 shows the phase velocities $C_{i p}(j=1,2)$, the attenuation coefficients $q_{j p}$ and the modulus and phase of the complex coefficients $c o_{j p}^{\prime}$ of the P1-wave and P2-wave in the saturated medium as a function of $\chi$. Similar variables for the $\mathrm{S}$-wave are shown in Fig. 4.

For limiting low values of $\chi$, the relative motion between both phases vanishes due to the high viscous coupling. This is reflected by the real and unit value of the coefficients $c o_{1 p}^{\prime}$ and $c o_{s}^{\prime}$. The saturated porous medium behaves as an equivalent undrained monophasic medium with a density equal to the mixture density and a low compressibility. The latter is due to the presence of pore water and reflected by a high value of the P-wave velocity $C_{p 0}=1745 \mathrm{~m} / \mathrm{s}$. The S-wave velocity is only weakly affected as it is only influenced by the change in density. It tends to the low frequency limit $C_{s 0}=235.9 \mathrm{~m} / \mathrm{s}$. In the low frequency limit, the ratio $s_{0}$ of $C_{s 0}$ and $C_{p 0}$ equals 0.135 and the corresponding undrained Poisson's ratio equals $v_{0}^{s}=0.49$. The attenuation coefficients $q_{1 p}$ and $q_{s}$ tend to zero. The P2-wave is diffusive.

For intermediate values of $\chi$, all waves are dispersive and attenuated. The absolute solid and fluid displacements are in phase in the P1-wave and out of phase in the P2-wave. Consequently, $q_{2 p}$ is larger than $q_{1 p}$ for all frequencies.

For limiting high values of $\chi$, the medium behaves as a non-dissipative poroelastic medium as the viscous coupling between both phases disappears and elastic as well as inertial coupling dominate the dispersion characteristics. The wave velocities $C_{j p}$ of both dilatational waves tend to the high-frequency limits $C_{p 1}=1824 \mathrm{~m} / \mathrm{s}$ and $C_{p 2}=303.7 \mathrm{~m} / \mathrm{s}$. The shear wave velocity also reaches its high frequency limit $C_{s 1}=249.78 \mathrm{~m} / \mathrm{s}$. The latter
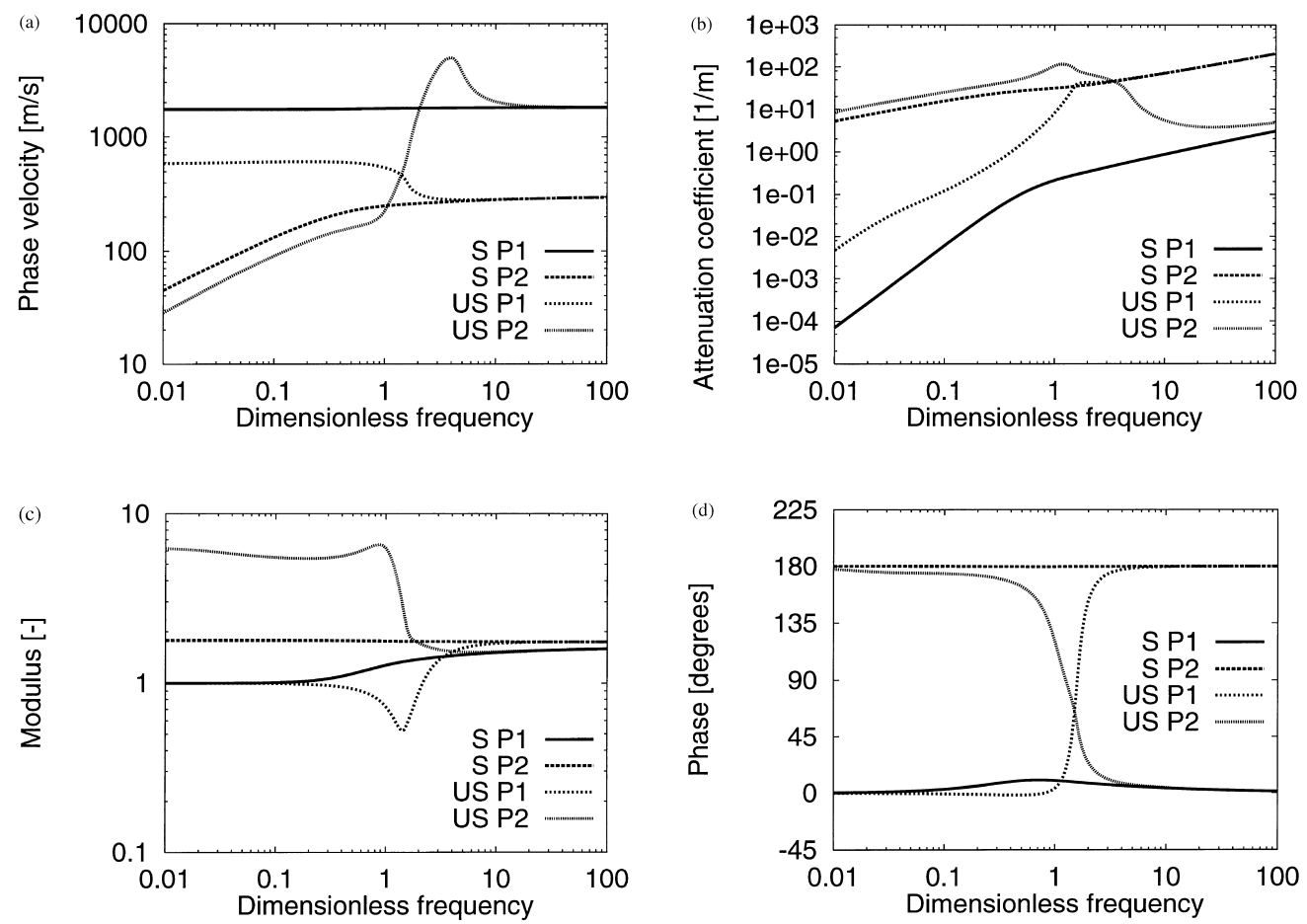

Fig. 3. (a) Phase velocities $C_{j p}$, (b) attenuation coefficients $q_{j p}$, (c) modulus, and (d) phase of the coefficients $c o_{j p}^{\prime}$ of the P1- and P2-wave in a saturated and an unsaturated $\left(g=0.001\right.$ and $R_{0}=0.5$ $\mathrm{mm})$ poroelastic medium as a function of the dimensionless frequency $\chi$. 

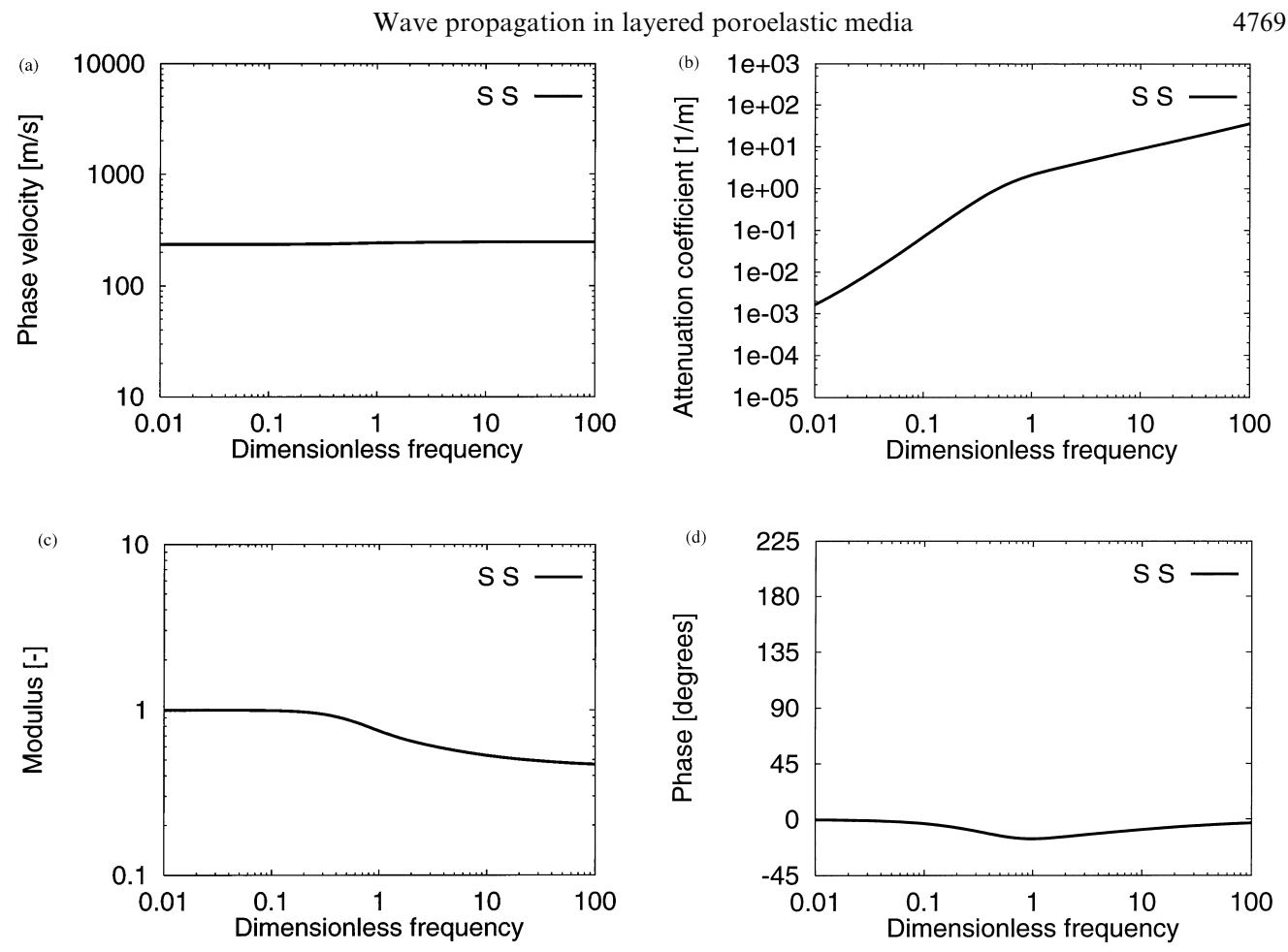

Fig. 4. (a) Phase velocity $C_{s}$, (b) attenuation coefficient $q_{s}$, (c) modulus, and (d) phase of the coefficient $c o_{s}^{\prime}$ of the $\mathrm{S}$-wave in a saturated poroelastic medium as a function of the dimensionless frequency $\chi$.

is smaller than the shear wave velocity in the drained solid skeleton due to the tortuosity of the pores.

5.2.3. Unsaturated poroelastic material. Considering next the unsaturated poroelastic material, it will first be demonstrated how a small amount of gas bubbles of initial radius $R_{0}$ and characterized by the gas fraction $g$, affects the pore fluid compressibility. Figure 5 shows the modulus and phase of the dimensionless fluid bulk modulus $K_{f} / K_{l}$ as a function of the dimensionless frequency $\chi$ for an unsaturated poroelastic medium with a gas fraction $g=0.001$ and bubble radii of $0.25,0.5$ and $1.0 \mathrm{~mm}$.

For low values of $\chi, K_{f}$ is only affected by the steady state compressibility of the gas bubbles as $K_{g}$ tends to $n_{p} p_{g 0}-\frac{2}{3}\left(\sigma / R_{0}\right)$ (Smeulders, 1992) and the dynamic bubble behaviour is of minor importance. For high values of $\chi$, the pore fluid acts as if there were no gas bubbles at all; $K_{g}$ tends to infinity and $K_{f}=K_{l} /(1-g)$. For intermediate values of $\chi$ however,
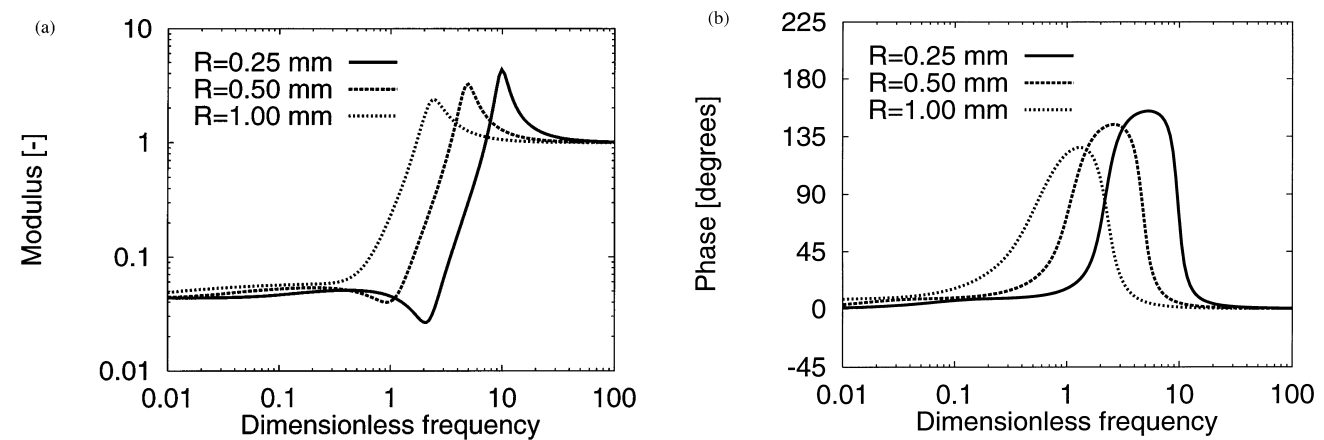

Fig. 5. (a) Modulus and (b) phase of the dimensionless fluid bulk modulus $K_{f} / K_{l}$ in an unsaturated poroelastic medium with gas fraction $g=0.001$ and bubble radii $R_{0}=0.25 \mathrm{~mm}, R_{0}=0.5 \mathrm{~mm}$ and $R_{0}=1.0 \mathrm{~mm}$ as a function of the dimensionless frequency $\chi$. 
the dynamic bubble behaviour is important. First, $K_{f}$ reaches a minimum. This high compressibility originates from a resonance phenomenon where the gas bubble volume changes in phase with the applied fluid pressure. The resonance frequency is inversely proportional to the bubble radius $R_{0}$ (Smeulders, 1992). At a higher frequency, an opposite phenomenon can be observed, i.e., the gas bubble volume increases when the pore fluid pressure is increased, resulting in an out of phase behaviour and an increase of $K_{f}$.

Because of the observed gas bubble resonance phenomenon, it is convenient to introduce in the unsaturated case a second dimensionless frequency $\bar{\omega}_{b}$ defined as the ratio of the excitation frequency $\omega$ and the gas bubble resonance frequency $\omega_{b}$. Both dimensionless frequencies $\chi$ and $\bar{\omega}_{b}$ are related by $\chi=\bar{\omega}_{b} \chi_{b}$, where $\chi_{b}$ is equal to the ratio of the gas bubble resonance frequency $\omega_{b}$ and the characteristic frequency $\omega_{0}$. A parametric study should account for a wide range of values for $\chi_{b}$. Here, however, only one bubble radius $\left(R_{0}=0.5\right.$ $\mathrm{mm}$ ) is considered for which $\omega_{b}=25,887 \mathrm{rad} / \mathrm{s}$ and $\chi_{b}=1.085$.

Figure 3 compares, as a function of $\chi$, the phase velocities $C_{j p}(j=1,2)$, the attenuation coefficients $q_{j p}$ and the modulus and phase of the complex coefficients $c o_{j p}^{\prime}$ of the P1-wave and P2-wave in a saturated and unsaturated poroelastic medium with gas fraction $g=0.001$ and bubble radius $R_{0}=0.5 \mathrm{~mm}$. The steady state compressibility of the gas bubbles is responsible for the decrease of both $C_{j p}$ at low $\chi$. The attenuation coefficients $q_{j p}$ in both waves increase. For higher frequencies, resonance and anti-resonance phenomena of the vibrating gas bubbles become important. $C_{1 p}$ decreases at the resonance frequency and tends to $C_{p 2}$ of the saturated poroelastic medium for high $\chi . C_{2 p}$ suddenly increases in the frequency range between the resonance and anti-resonance frequency of the gas bubbles, reaches a maximum at the latter frequency and tends to $C_{p 1}$ of the saturated poroelastic medium for high $\chi \cdot q_{2 p}$ reaches a maximum at the resonance frequency of the gas bubbles. In the high frequency regime, the roles of both $\mathrm{P}$-waves change, as the phase velocity and attenuation coefficient of the P1-wave tend to the corresponding values of the P2-wave of the non-dissipative poroelastic medium and vice versa.

\subsection{Results in the wavenumber-frequency domain}

In order to illustrate the effect of a moving water table and the presence of air bubbles on wave propagation in an axisymmetric halfspace consisting of sand of Mol, the following six cases with increasing degree of saturation are considered: (a) a dry halfspace (D); (b) a dry layer with thickness $H$ on an unsaturated halfspace (D-US); (c) a dry layer with thickness $H$ on a saturated halfspace (D-S); (d) an unsaturated halfspace (US); (e) an unsaturated layer with thickness $H$ on a saturated halfspace (US-S); and (f) a saturated halfspace (S).

Each halfspace is subjected to a unit vertical traction $T_{z}(r, z=0, t)=\delta(r) \delta(t)$ at the free surface, where $\delta(\cdot)$ denotes the Dirac delta function. This function has a unit spectral content in the wavenumber and frequency domain so that the solution is not influenced by the spatial and temporal distribution of the loading. Halfspace and layer elements are used in an exact stiffness formulation to solve the governing wave equations in the wavenumberfrequency domain with generalized coordinates $\left(k_{r}, z, \omega\right)$. The $z$-axis is pointing downwards.

For the calculations in the wavenumber-frequency domain, we have used a low value $\beta_{s}^{s}=\beta_{p}^{s}=0.001$ for the material damping ratios. This low value is not related to the material damping that is actually observed in real soils. It rather has a mathematical meaning in a sense that it removes the surface wave pole(s) from the real horizontal wavenumber axis (Apsel and Luco, 1983; Kundu and Mal, 1985). At the same time, it is low enough to reduce the influence of material damping on the computational results at high frequencies. In real soils however, material damping is higher. A typical value for $\beta_{s}^{s}$ and $\beta_{p}^{s}$ in sandy soils is equal to 0.02 , as revealed by in situ experiments such as a down-hole survey (Kokusho, 1987). This realistic value will be used for the subsequent calculations in the space-time domain.

For the six cases considered, Fig. 6 shows the modulus of the vertical solid skeleton displacement $\tilde{u}_{z}^{s}\left(k_{r}, z=0, \omega\right)$ at the free surface as a function of the dimensionless horizontal wavenumber $\bar{k}_{r}$ and the dimensionless frequency $\chi$. The dimensionless horizontal wavenumber $\bar{k}_{r}$ is defined as $k_{r} C_{s} / \omega=C_{s} / C$ where $C_{s}$ is the shear wave velocity of the drained 

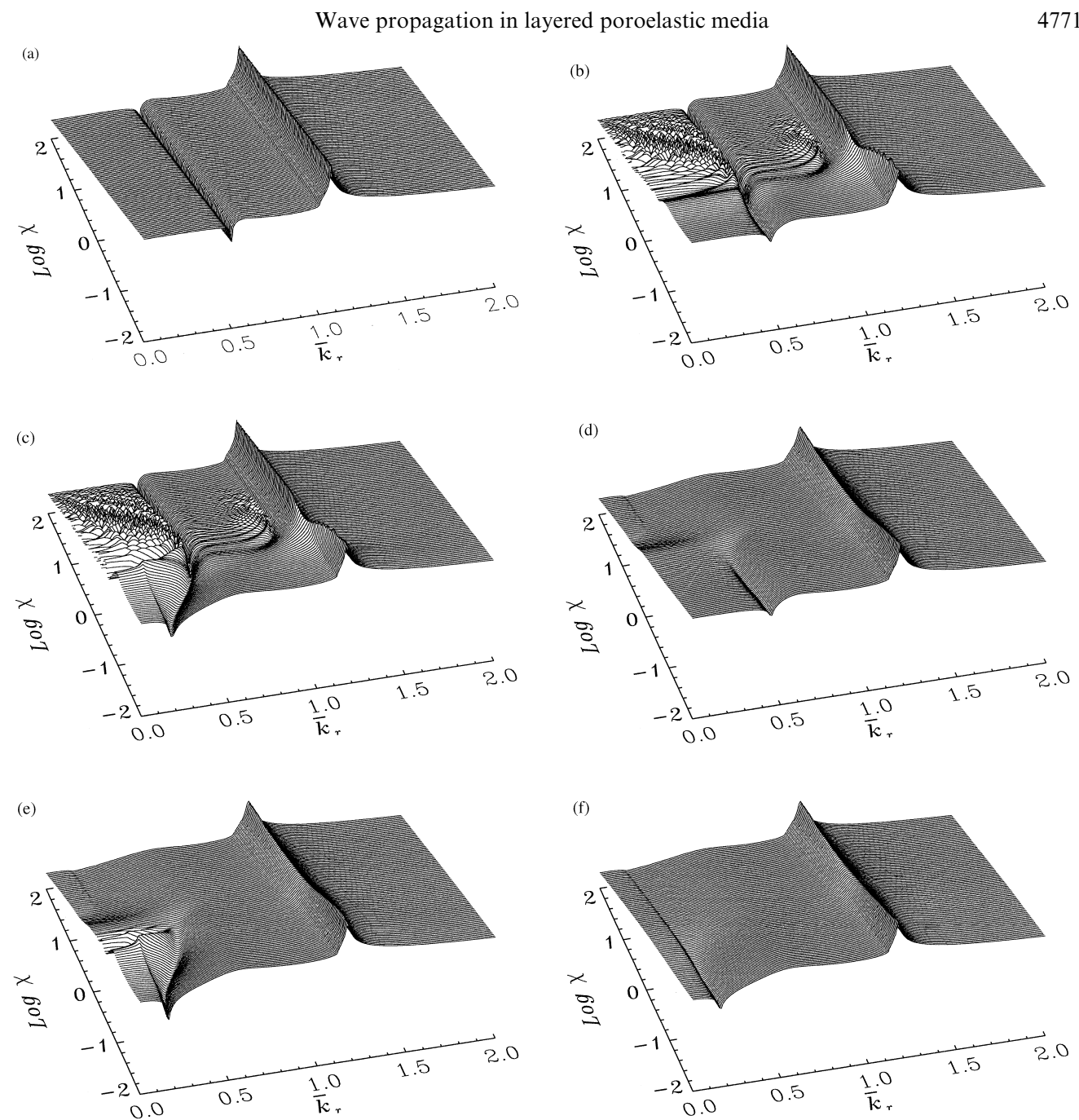

Fig. 6. Logarithm of the modulus of the vertical solid skeleton displacement at the free surface as a function of the dimensionless frequency $\chi$ and the dimensionless horizontal wavenumber $\bar{k}_{r}$ for the six cases considered. (a) D. (b) D-US. (c) D-S. (d) US. (e) US-S. (f) S.

porous medium and $C$ is the phase velocity. Both the $\chi$-axis and the vertical axis are on a logarithmic scale. $\chi$ varies between 0.01 and 100.0. The $\bar{k}_{r}$-axis is linear and $\bar{k}_{r}$ varies between 0 and 2.

In the following subsections, general observations will be made regarding the response in the wavenumber-frequency domain for the six cases. Further conclusions will be drawn when results in the space-time domain are presented for a specific frequency content of the loading.

5.3.1. Dry halfspace. The response of a dry halfspace does not depend on the dimensionless frequency $\chi$ that has been defined in the context of saturated media. The overall frequency dependence of the response is restricted to a decreasing response for increasing frequency due to material damping. As the amount of material damping is small $(0.1 \%)$, this effect will only be important at high frequencies.

For $\bar{k}_{r}=0$, corresponding to the one-dimensional case, the phase velocity $C$ is infinite and the P-and S-wave are uncoupled and propagate in the $z$-direction (Fig. 6(a)). For $\vec{k}_{r}>0$, the phase velocity $C$ is finite and the free surface condition couples the P- and Swave, that are both propagative in $r$ and $z$. The P-wave becomes inhomogeneous with exponential decay in the $z$-direction for $\bar{k}_{r} \geqslant s\left(C \leqslant C_{p}\right)$, where the ratio $s$ equals 0.5 in this example. A similar remark can be made for the S-wave if $\bar{k}_{r} \geqslant 1\left(C \leqslant C_{s}\right)$. For $\bar{k}_{r} \simeq 1.07$, a 
peak according to the nondispersive Rayleigh (R-) wave appears (Raleigh, 1887). Due to the introduction of a small amount of material damping, the Rayleigh wave pole has been moved away from the real wavenumber axis and the response remains finite at all frequencies.

5.3.2. Saturated halfspace. It is instructive to discuss the case of the saturated halfspace before layering or air bubbles are introduced. The dispersive character of the P1-wave and the R-wave in a saturated poroelastic medium can be observed from Fig. 6(f).

For low $\chi$, the saturated medium behaves as an undrained monophasic mixture. This explains the high velocity of the P1-wave, as mentioned before. The P1-wave becomes inhomogeneous in $z$ if $\bar{k}_{r}$ is larger than $C_{s} / C_{p 0}$. As the ratio $C_{s 0} / C_{p 0}$ of the low frequency body wave velocities is equal to 0.135 and the ratio $C_{s} / C_{s 0}$ is equal to 1.113 , a corresponding dip in the displacement modulus can be observed at $\bar{k}_{r}=0.15$. The P2-wave is diffusive. The S-wave velocity is only weakly affected as it is only influenced by the change in density. The secular equation that governs the surface wave behaviour in a saturated poroelastic medium follows immediately from the solution of a transcendental eigenvalue problem (52), where the stiffness matrix $\widetilde{\mathbf{K}}^{S}$ reduces to the saturated halfspace element stiffness matrix. This has been studied in much detail by Deresiewicz (1962) and Jones (1961). For low $\chi$, the solution of the secular equation is $\bar{k}_{r}=\left(k_{r} C_{s 0} / \omega\right)\left(C_{s} / C_{s 0}\right)=1.048 \times 1.113=1.166$. The surface wave velocity in the saturated poroelastic halfspace is lower than in the dry halfspace due to the high value of the mixture density. The lower compressibility results in smaller displacements.

For limiting high $\chi$, the medium behaves as a non-dissipative poroelastic solid. The Swave and R-wave are only affected by the tortuosity of the pores. The elastic as well as inertial coupling result in considerable influence on P-wave propagation.

For intermediate $\chi$, dispersion and attenuation of all waves affect the vertical displacement at the surface.

5.3.3. Unsaturated halfspace. When compared to the saturated case, the presence of air bubbles in an unsaturated halfspace only slightly influences the R-wave propagation (Fig. 6(d)), as the R-wave velocity is mainly influenced by the shear modulus and the density. The S-wave propagation is not affected by the presence of air bubbles. However, considerable influence of a small amount of air bubbles on P-wave propagation can be observed. For low $\chi$, the P-wave velocity is much lower than in the saturated case due to the high compressibility of the air bubbles and is only slightly higher than in the dry case. For high $\chi$, the presence of air bubbles is of minor importance and the response tends to the response of the saturated halfspace. For intermediate $\chi$, however, the transition behaviour in the vicinity of the resonance and anti-resonance frequency of the air bubbles can clearly be observed.

5.3.4. Dry layer on a saturated halfspace. Next, we consider the case of a dry layer on a saturated halfspace (Fig. 6(c)). This problem clarifies the influence of a moving ground water table due to seasonal variations or temporal changes as they may be caused by an artificial lowering of the ground water table during construction works. This case has also been studied by Philippacopoulos $(1987 ; 1988 b)$.

Besides the dimensionless frequencies $\chi$ and $\bar{\omega}_{b}$ introduced in the context of saturated and unsaturated media, it is convenient to introduce here a third dimensionless frequency $\bar{\omega}_{c o}$, defined as the ratio of the excitation frequency $\omega$ and the first cut-off frequency $\omega_{c o}$ of the dry layer built in at its base. The latter is equal to $2 \pi C_{s} / 4 H$ for horizontal motion and $2 \pi C_{p} / 4 H$ for vertical motion, with $H$ the depth of ground water table. At these resonance frequencies, the height of the layer equals the quarter wavelength. As the loading is vertical, we will use the vertical resonance frequency in the following. Both dimensionless frequencies $\chi$ and $\bar{\omega}_{c o}$ are related by $\chi=\bar{\omega}_{c o} \chi_{c o}$, where $\chi_{c o}$ is equal to the ratio of the first cut-off frequency $\omega_{c o}$ of the layer built in at its base and the characteristic frequency $\omega_{0}$. A parametric study should account for a wide range of values for $\chi_{c o}$. In the present example, 
however, only one value for the depth of the water table $(H=0.25 \mathrm{~m})$ has been considered. The corresponding vertical cut-off frequency equals $\omega_{c o}=3302 \mathrm{rad} / \mathrm{s}$ and $\chi_{c o}=0.1384$.

The presence of a ground water table at a depth $H$ below the free surface results in a high contrast in compressibility between the dry layer and the underlying saturated halfspace due to the low compressibility of the pore fluid. The influence of the layering introduced by the ground water table depends on the dimensionless frequency $\bar{\omega}_{c o}$, or $\chi$ when $\chi_{c o}$ is fixed. For limiting low and high values of $\chi$, the medium behaves like a saturated (Fig. 6(f)) or a dry (Fig. 6(a)) halfspace, respectively. The first surface wave is dispersive and its phase velocity varies from the R-wave velocity in a saturated halfspace to that of a dry halfspace for increasing $\chi$. At $\bar{\omega}_{c o}=1,3, \ldots$, higher surface modes appear. For $\bar{\omega}_{c o}$ larger than one, the solution becomes oscillatory for low values of $\bar{k}_{r}$.

5.3.5. Dry layer on an unsaturated halfspace. The response of a dry layer on an unsaturated halfspace depends on the dimensionless frequencies $\chi, \bar{\omega}_{c o}$ and $\bar{\omega}_{b}$ as introduced before. In this example, the permeability $(k=0.0001 \mathrm{~m} / \mathrm{s})$, the depth of the ground water table $(H=0.25 \mathrm{~m})$ and the gas bubble radius $(R=0.5 \mathrm{~mm})$ are fixed. The corresponding values of the characteristic frequency $\omega_{0}$, the cut-off frequency $\omega_{c o}$ of the layer built in at its base and the gas bubble resonance frequency $\omega_{b}$ are fixed so that only the effect of the excitation frequency $\omega$ will be visible in the numerical results.

Figure 6(b) demonstrates that the presence of an unsaturated halfspace with a small amount of air bubbles in the pores at a depth $H$ below the free surface introduces layering of which the influence depends on the dimensionless frequency $\bar{\omega}_{c o}$, or $\chi$ when $\chi_{c o}$ is fixed. For limiting low and high values of $\chi$, the medium behaves as an unsaturated (Fig. 6(d)) or a dry (Fig. 6(a)) halfspace. The first surface wave is dispersive and its phase velocity varies between the R-wave velocity in an unsaturated halfspace and that of a dry halfspace. The R-wave has almost the same characteristics as in the case of a dry layer on a saturated halfspace. When compared to the case of a dry layer on a saturated halfspace (Fig. 6(c)), however, the propagation of $\mathrm{P}$-waves is largely influenced by the presence of a small amount of air bubbles in the underlying halfspace, due to the high compressibility of the air bubbles at low frequencies and the resonance and anti-resonance behaviour of air bubbles at intermediate frequencies.

5.3.6. Unsaturated layer on a saturated halfspace. As can be observed from Fig. 6(e), the presence of a small amount of air bubbles in the top layer of a saturated halfspace considerably affects the propagation of P-waves. The response tends to the response of a saturated halfspace (Fig. 6(f)) in the low frequency range and the response of an unsaturated halfspace (Fig. 6(d)) in the high frequency range.

\subsection{Results in the time domain}

Next, the transient response of the six cases is calculated due to an impulsive point force $T_{z}(r, z=0, t)=\delta(r) R(t)$ at the free surface. $R(t)$ is a Ricker wavelet defined as $R(t)=\left(2 \bar{t}^{2}-1\right) \exp \left(-\bar{t}^{2}\right)$ where $\bar{t}=\pi\left(t-t_{s}\right) / T_{d}$ is a dimensionless time, $T_{d}$ the dominant period, and $t_{s}$ a shift in time. In this example, these parameters are equal to $T_{d}=2.5 \mathrm{~ms}$ and $t_{s}=5.0 \mathrm{~ms}$. Figure 7 shows the time history and frequency content of this Ricker wavelet. The spectral content has been calculated with an FFT algorithm with period $T=0.064 \mathrm{~s}$ and time step $\Delta t=0.25 \mathrm{~ms}$ and reveals a dominant frequency at $400 \mathrm{~Hz}$.

The inverse integral transformations from $k_{r}$ to $r$ are evaluated numerically by means of an adaptive generalized Filon method (Frazer and Gettrust, 1984). The behaviour of the part $f\left(k_{r}, z, \omega\right)$ of the integrand is well described in function of the dimensionless wave number $\bar{k}_{r}$. A target value of the dimensionless quadrature step $\Delta \bar{k}_{r}$ is specified. A particular value of $\Delta \bar{k}_{r}$ defines an increasing spatial window length $L$ for decreasing frequency $\omega$. However, the choice of the quadrature step is not governed by a requested absolute and/or relative accuracy over the whole integration interval in the present implementation. Instead, $\Delta \bar{k}_{r}$ is allowed to decrease or increase with a factor of 16, depending on an integration error, estimated as the contribution of the fourth order term to the integral in the integration interval under consideration. The upper bound restriction on $\Delta \bar{k}_{r}$ is relaxed for $\bar{k}_{r}>1.5$ 

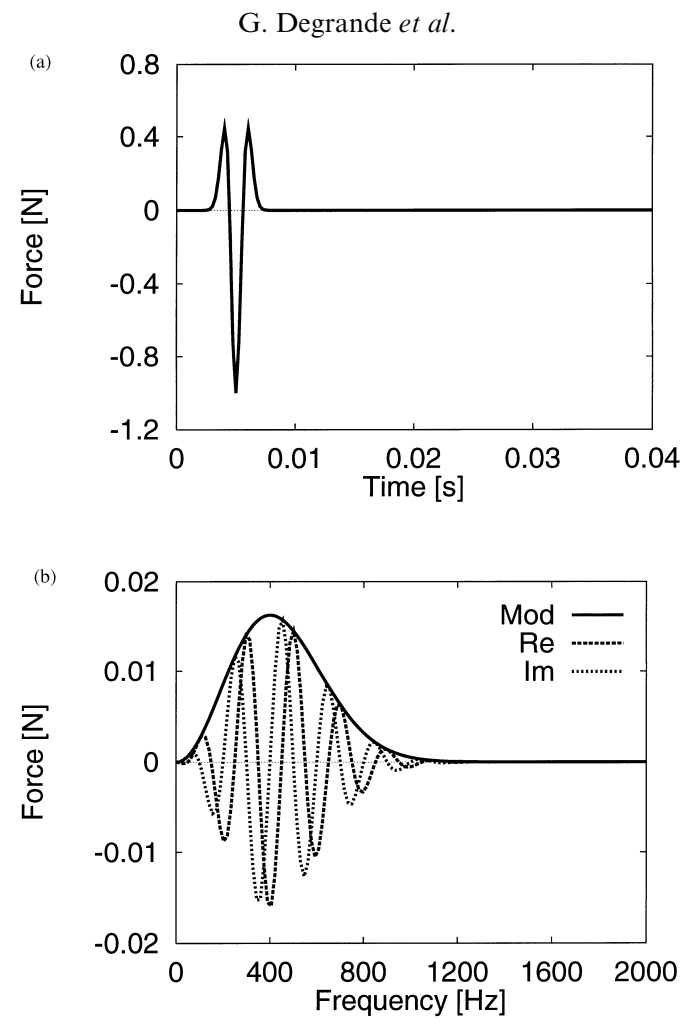

Fig. 7. (a) Time history and (b) frequency content of the Ricker wavelet with $T_{d}=2.5 \mathrm{~ms}$ and $t_{s}=5.0 \mathrm{~ms}$.

beyond which the integrand is known to be monotonic. A target value of $\Delta \bar{k}_{r}=0.01$ was chosen in this example. The total integration interval is truncated at $\bar{k}_{r}^{\max }$. A particular value of $\bar{k}_{r}^{\max }$ defines a decreasing spatial resolution for increasing frequency $\omega$. In this example, the integration is continued up to $\bar{k}_{r}^{\max }=320.0$, a value based on experience rather than on a criterium based on requested accuracy.

Figure 8 shows the time history of the vertical displacement $u_{z}^{s}(r, z=0, t)$ in the solid skeleton at the free surface at different distances $r=r_{0}+(i-1) \Delta r$ from the source with $r_{0}=2.5 \mathrm{~m}, \Delta r=0.25 \mathrm{~m}$ and $i=1, \ldots, 11$ for the six cases considered. Note that $2 \%$ of material damping in dilatational and rotational deformation has been introduced, a realistic value for material attenuation in sandy soil. Figure 8(a) shows the time histories in the dry halfspace. These results will be treated as a reference and are repeated as dashed lines on the other figures. The observations that can be made in the space-time domain follow immediately from earlier observations in the wavenumber-frequency domain. Therefore, it is interesting to consider Fig. 6 simultaneously. The center frequency of the loading is equal to $400 \mathrm{~Hz}$, which corresponds to a dimensionless frequency $\chi=0.105$. A complete parametric study should include a wide range of input wavelets with different dominant frequency.

5.4.1. Dry halfspace. In the dry halfspace, the arrival of the P-wave and the R-wave, with a much higher amplitude, can clearly be observed (Fig. 8(a)). The S-wave arrives just before the R-wave. The amplitudes of these waves are attenuated by material damping and geometrical spreading. As the latter is much higher for body waves at the surface than for surface waves, the $\mathrm{R}$-wave dominates the response in the far field.

5.4.2. Dry layer on an unsaturated halfspace. The water table is raised next up to a depth $H$ below the free surface while the underlying halfspace is unsaturated. Due to the high compressibility of the air bubbles in the pores of the unsaturated halfspace, the Pwave velocity in the unsaturated halfspace is only slightly larger than in the dry layer. The 

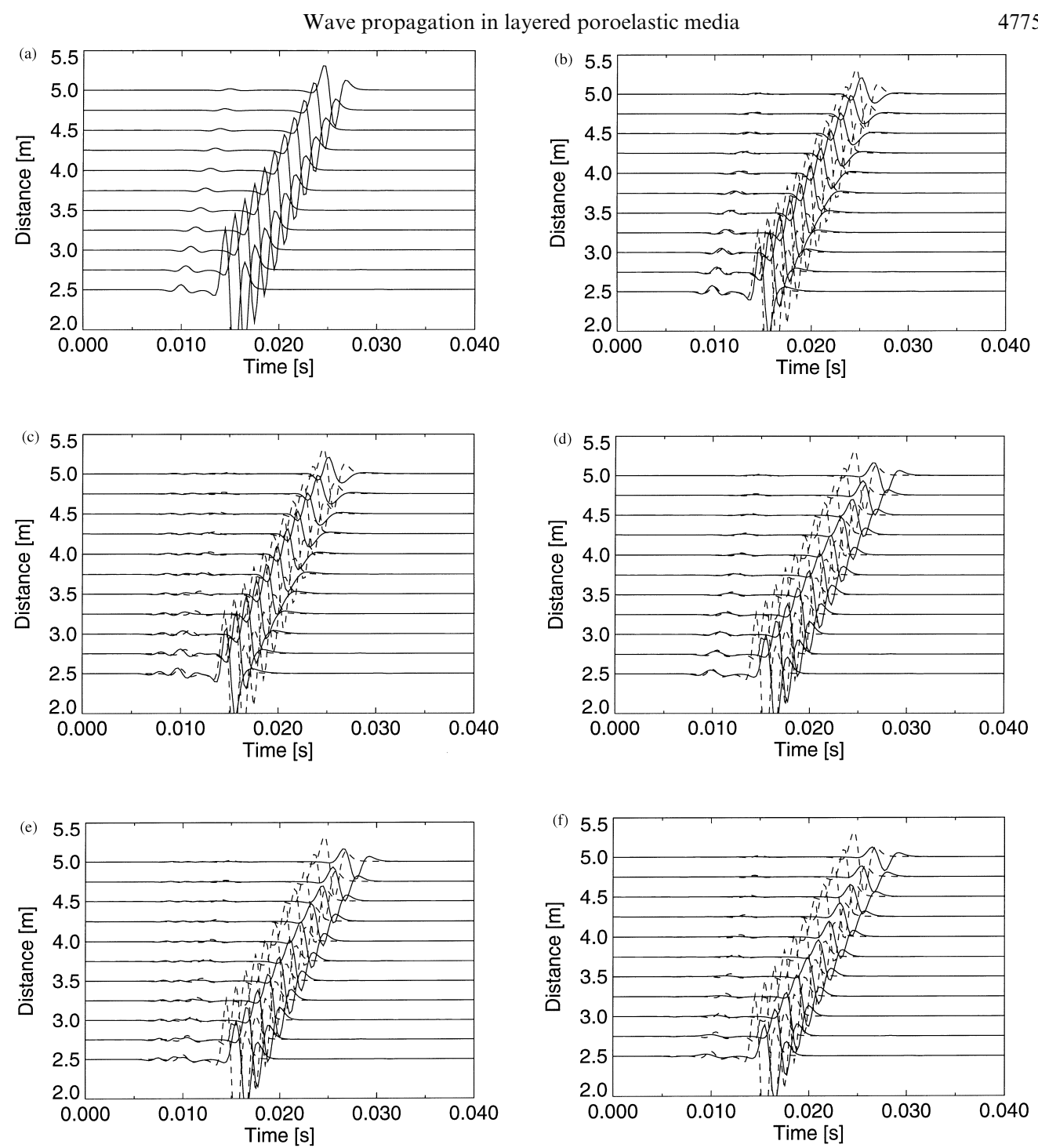

Fig. 8. Time history of the vertical solid skeleton displacement at the free surface (solid line) for various source-receiver distances for the six cases considered. The dashed line denotes the time history on top of the dry halfspace which is considered as the reference case. (a) D. (b) D-US. (c)

D-S. (d) US. (e) US-S. (f) S.

resulting contrast in $\mathrm{P}$-wave velocity is small and $\mathrm{P}$-wave propagation is only weakly affected (Fig. 8(b)). Some dispersion can be observed.

In the frequency range of interest, the R-wave is dispersive. The phase velocity of the first surface mode varies between the R-wave velocity of an unsaturated halfspace and a dry halfspace. Therefore, low frequency components travel with a lower velocity than components at higher frequencies. The amplitudes of the R-wave components are smaller than in the dry halfspace, due to the slightly lower compressibility of the underlying unsaturated material and the larger attenuation.

5.4.3. Dry layer on a saturated halfspace. When the halfspace below the dry layer is fully saturated, the low compressibility of the pore fluid results in a high contrast between the compressibility of the layer and the underlying halfspace. In the frequency range of interest, this gives rise to wave refraction and resonance phenomena at the first vertical eigenfrequency of the layer built in at its base, which is clearly observable from the P-wave arrivals in Fig. 8(c). 
The $\mathrm{R}$-wave is dispersive and the phase velocity of the first surface mode varies between the R-wave velocity of a saturated halfspace and a dry halfspace. The former is almost the same as the R-wave velocity in the unsaturated halfspace, as it is mainly influenced by the shear modulus of the drained solid skeleton and the mixture density, characteristics which are unaffected by the presence of a small amount of air bubbles. When compared to the previous case of the dry layer on an unsaturated halfspace (Fig. 8(b)), the low compressibility of the underlying halfspace does not considerably affect the displacement amplitudes at the free surface. When compared to the dry halfspace however, lower amplitudes can be observed since the compressibility of the unsaturated material is still lower than the compressibility of the dry material.

5.4.4. Unsaturated halfspace. The ground water table is subsequently raised up to the level of the free surface. The halfspace in unsaturated. In the frequency range of interest, the propagation of the P1-wave is affected by the high compressibility of the air bubbles (Fig. 8(d)). The P1-wave velocity is slightly higher than the P-wave velocity in the dry material. As the frequency content of the loading is well beyond the resonance and antiresonance frequency of the air bubbles, these phenomena are not important here.

The $\mathrm{R}$-wave propagation is influenced throughout the whole frequency range, resulting in a slower R-wave with a lower amplitude than in the dry case. As the compressibility is higher than in the saturated case, the amplitude is larger than in the saturated case (Fig. $8(\mathrm{f})$ ).

5.4.5. Unsaturated layer on a saturated halfspace. The unsaturated halfspace is fully saturated up to a depth $H$ below the free surface. The propagation of P-waves is strongly affected. Due to the presence of a small amount of air bubbles in the top layer, a contrast in compressibility is introduced between the unsaturated layer and the underlying saturated halfspace, resulting in resonance phenomena at the first vertical resonance frequency of the unsaturated layer built in at its base (Fig. 8(e)). This result demonstrates that partial saturation of the top layer of a saturated halfspace may have important effects on P-wave propagation.

The $\mathrm{R}$-wave velocity is weakly dispersive and the $\mathrm{R}$-wave velocity varies from the $\mathrm{R}$ wave velocity in a saturated halfspace to the R-wave velocity in an unsaturated halfspace. The influence of the compressibility on the R-wave velocity is small however, whereas the influence on the displacement amplitudes is higher. Due to the higher compressibility of the top layer, the resulting amplitudes are higher than in the saturated halfspace (Fig. 8(f)), but lower than in an unsaturated halfspace (Fig. 8(d)).

5.4.6. Saturated halfspace. When the halfspace is fully saturated, a fast P1-wave and a slower dispersive R-wave can be observed (Fig. 8(f)). Due to the low compressibility of the material, the resulting displacement amplitudes are lower than in the dry halfspace.

\section{CONCLUSION}

An exact stiffness formulation has been presented to study harmonic and transient wave propagation in multilayered dry, saturated and unsaturated isotropic poroelastic media. The description of wave propagation and attenuation in unsaturated porous media incorporates the dynamic gas bubble behaviour. The unsaturated case is restricted to small amounts of gas in the pores of the saturated medium so that Smeulders' extension of Biot's poroelastic theory can be used. Consequently, an exact stiffness formulation for saturated porous media can be simply modified to incorporate the unsaturated case.

The versatility of the method has been demonstrated by a numerical example where the effect of partial saturation and a moving water table on harmonic and transient wave propagation in an axisymmetric layered halfspace has been treated. The response of the media is discussed in terms of three dimensionless frequencies $\chi, \bar{\omega}_{b}$ and $\bar{\omega}_{c o}$, defined as the 
ratio of the excitation frequency $\omega$ to the characteristic frequency $\omega_{0}$, the resonance frequency $\omega_{b}$ of the air bubble (unsaturated case), and the cut-off frequency $\omega_{c o}$ of the top layer (water table below the free surface), respectively.

The effect of an increasing degree of saturation of a halfspace on the propagation of harmonic waves could clearly be illustrated in the wavenumber-frequency domain, despite our limitation to one particular value of the permeability $k$, the air bubble radius $R_{0}$, and the depth $H$ of the ground water table. A similar conclusion is true for results obtained in the space-time domain after evaluation of the inverse integral transformations. These results are also influenced by the particular spectral content of the loading in the wavenumber and frequency domain. For the low frequency loading applied in this example, the effects of partial saturation and a moving ground water table on $\mathrm{R}$-wave propagation were noticeable but small. P-wave propagation however is importantly influenced when a considerable contrast in compressibility is introduced at a certain depth $H$ below the free surface. This is the case for a dry or unsaturated layer on a saturated halfspace. The latter means that the presence of air bubbles in a top layer of a saturated halfspace may considerably affect P-wave propagation.

\section{REFERENCES}

Achenbach, J. and Epstein, H. (1967) Dynamic interaction of a layer and a half-space. Journal of the Engineering Mechanics Division, Proceedings of the ASCE 93(EM5), 27-42.

Apsel, R. and Luco, J. (1983) On the Green's functions for a layered half-space. Part II. Bulletin of the Seismological Society of America 73, 931-951.

Atkin, R. (1968) Completeness theorems for linearized theories of interacting continua. Quarterly Journal of Mechanics and Applied Mathematics XXI(Pt a), 171-193.

Auriault, J.-L., Borne, L. and Chambon, R. (1985) Dynamics of porous saturated media, checking of the generalized law of Darcy. Journal of the Acoustical Society of America 77, 1641-1650.

Bateman, H. (1954) Tables of Integral Transforms. California Institute of Technology, Bateman Manuscript Project, McGraw-Hill, New York.

Biot, M. A. (1956) Theory of propagation of elastic waves in a fluid saturated porous solid. I. Low-frequency range. Journal of the Acoustical Society of America 28, 168-178.

Bouchon, M. and Aki, K. (1977) Discrete wave-number representation of seismic-source wave fields. Bulletin of the Seismological Society of America 67(2), 259-277.

Bougacha, S., Tassoulas, J. and Roesset, J. (1993) Analysis of foundations on fluid-filled poroelastic stratum. Journal of the Engineering Mechanics Division, Proceedings of the ASCE 119(8), 1632-1648.

Bourbié, T., Coussy, O. and Zinszner, B. (1987) Acoustics of Porous Media. Gulf Publishing Company, Editions Technip.

Bowen, R. and Reinicke, K. (1978) Plane progressive waves in a binary mixture of linear elastic materials. Journal of Applied Mechanics, Transactions of the ASME 45, 493-499.

Coussy, O. (1995) Mechanics of Porous Continua. John Wiley and Sons, Chichester.

Degrande, G. (1992) A spectral and finite element method for wave propagation in dry and saturated poroelastic media. Ph.D. thesis, Department of Civil Engineering, Katholieke Universiteit Leuven.

Degrande, G. and De Roeck, G. (1992) A spectral element method for two-dimensional wave propagation in horizontally layered saturated porous media. Computers and Structures 44(4), 717-728.

Degrande, G. and De Roeck, G. (1993) An absorbing boundary condition for wave propagation in saturated poroelastic media-Part I : Formulation and efficiency evaluation. Soil Dynamics and Earthquake Engineering 12(7), 411-421

Deresiewicz, H. (1962) The effect of boundaries on wave propagation in a liquid-filled porous solid : IV. Surface waves in a half-space. Bulletin of the Seismological Society of America 52(3), 627-638.

Deresiewicz, H. and Skalak, R. (1963) On uniqueness in dynamic poroelasticity. Bulletin of the Seismological Society of America 53(4), 783-788.

Draelants, G. (1994) Dispersie van oppervlaktegolven in een horizontaal gelaagde halfruimte. Master's thesis, Department of Civil Engineering, Katholieke Universteit Leuven.

Eringen, A and Suhubi, E. (1975) Elastodynamics, Volume 2, Linear Theory. Academic Press, New York, USA.

Frazer, L. and Gettrust, J. (1984) On a generalization of Filon's method and the computation of the oscillatory integrals of seismology. Geophysical Journal of the Royal Astrological Society 76, 461-481.

Garg, S., Nayfeh, A. H. and Good, A. (1974) Compressional waves in fluid-saturated elastic porous media. Journal of Applied Physics 45(5), 1968-1974.

Haskell, N. (1953) The dispersion of surface waves on multilayered media. Bulletin of the Seismological Society of America 73, 17-43.

Johnson, D., Koplik, J. and Dashen, R. (1987) Theory of dynamic permeability and tortuosity in fluid-saturated porous media. Journal of Fluid Mechanics 176, 379-402.

Jones, J. (1961) Rayleigh waves in a porous, elastic, saturated solid. Journal of the Acoustical Society of America 33(7), 959-962.

Kausel, E. and Roesset, J. (1981) Stiffness matrices for layered soils. Bulletin of the Seismological Society of America 71(6), 1743-1761.

Kokusho, T. (1987) In-situ dynamic soil properties and their evaluations. Proceedings of the 8th Asian Regional Conference on Soil Mechanics and Foundation Engineering, Vol. 2, pp. 215-240. Kyoto, Japan. 
Kundu, T. and Mal, A. (1985) Elastic waves in a multilayered solid due to a dislocation source. Wave Motion 7 , $459-471$.

Luco, J. and Apsel, R. (1983) On the Green's functions for a layered half-space. Part I. Bulletin of the Seismological Society of America 4, 909-929.

Paul, S. (1976a) On the displacements produced in a porous elastic halfspace by an impulsive line load (nondissipative case). Pure and Applied Geophysics 114, 605-614.

Paul, S. (1976b) On the disturbance produced in a semi-infinite poroelastic medium by a surface load. Pure and Applied Geophysics 114, 615-627.

Philippacopoulos, A. (1987) Waves in a partially saturated layered halfspace: analytic formulation. Bulletin of the Seismological Society of America 77(5), 1838-1853.

Philippacopoulos, A. (1988a) Lamb's problem for fluid-saturated, porous media. Bulletin of the Seismological Society of America 78(2), 908-923.

Philippacopoulos, A. (1988b) Waves in partially saturated medium due to surface loads. Journal of the Engineering Mechanics Division, Proceedings of the ASCE 114(10), 1740-1759.

Rajapakse, R. and Senjuntichai, T. (1995) Dynamic response of a multi-layered poroelastic medium. Earthquake Engineering and Structural Dynamics 24, 703-722.

Rayleigh, J. (1887) On waves propagated along the plane surface of an elastic solid. Proceedings of the London Mathematical Society 17, 4-11.

Smeulders, D. (1992) On wave propagation in saturated and partially saturated porous media. Ph.D. thesis, Technische Universiteit Eindhoven.

Smeulders, D., Eggels, R. and Van Dongen, M. (1992) Dynamic permeability: reformulation of theory and new experimental and numerical data. Journal of Fluid Mechanics 245, 211-227.

Smeulders, D., van Dongen, M. and De la Rosette, J. (1992) Waves in partially saturated porous media. Transport in Porous Media 9(1-2), 25-37.

Thooft, K. (1992) Theoretisch experimentele beschrijving van het spannings vervormingsgedrag van onsamenhangende gronden onder uitwendige dynamische belastingen, in het bijzonder bij grote verglijdingen. Ph.D. thesis, Department of Civil Engineering, Universiteit Gent.

Van den Broeck, P., Degrande, G. and De Roeck, G. (1993) Wave propagation in axisymmetric dry elastic media : theory and experimental verification. Report to IWONL, Department of Civil Engineering, Katholieke Universiteit Leuven. IWONL Research Grant CI 1/4-7672/091.

Van Impe, W. (1981) Studie van het vervormingsgedrag van Molzand onder cyclisch wisselende belastingen Ph.D. thesis, Department of Civil Engineering, Rijksuniversiteit Gent.

Wolfram, S. (1991) Mathematica. A system for doing mathematics by computer, 2nd edn. Addison-Wesley, Reading, MA.

Xu, P.-C. and Mal, A. (1985) An adaptive integration scheme for irregularly oscillatory functions. Wave Motion 7, 235-243.

Xu, P.-C. and Mal, A. (1987) Calculation of the inplane Green's functions for a layered viscoelastic solid. Bulletin of the Seismological Society of America 77(5), 1823-1837.

Yoon, Y.-W. (1992) Static and dynamic behaviour of crushable and non-crushable sands. Ph.D. thesis, Department of Civil Engineering, Universiteit Gent. 